\title{
COMPUTING THE INFINITESIMAL INVARIANTS ASSOCIATED TO DEFORMATIONS OF SUBVARIETIES
}

\author{
RANDALL F. WesthofF
}

The purpose of this article is to study and describe a method for computing the infinitesimal invariants associated to deformations of subvarieties. An interpretation of the infinitesimal invariant of normal functions as a pairing similar to the infinitesimal Abel-Jacobi mapping is given. The computation of both invariants for certain forms is then reduced to a residue computation at a finite number of points of the subvariety. Applications of this technique include a nonvanishing result for the infinitesimal Abel-Jacobi mapping leading to finiteness results for low degree rational curves on complete intersection threefolds with trivial canonical bundle and a generalization of a formula of Voisin for the infinitesimal invariant of certain normal functions.

\section{Introduction.}

The problem of understanding the subvarieties of a given projective variety is one of the central problems in algebraic geometry. If $Y$ is a subvariety of a projective variety $X \subseteq \mathbb{P}^{m}$ and $Y$ is nontrivial in the sense that it is the not the complete intersection of $X$ with another subvariety of $\mathbb{P}^{m}$, then in many situations one expects the deformations of $Y$ in $X$ to "generate" some of the cohomology of $X$. The cohomology generated by a subvariety as it deforms is measured by Abel-Jacobi mappings, normal functions and their infinitesimal variants. Understanding the degree to which deformations of a subvariety generate the cohomology of a variety can yield information about the structure of the family of all such subvarieties. (i.e. dimension, smoothness, etc.)

We begin with a brief discussion of Abel-Jacobi mappings. Let $X$ be a smooth projective variety of dimension $n$ and let $F$ be a smooth projective variety parametrizing a family of subvarieties of dimension $d$ on $X$. Let $E=\{(Y, x) \in F \times X: x \in Y\}$ and let $p: E \longrightarrow F$ and $q: E \longrightarrow X$ be the natural projections. Then the "cohomological" Abel-Jacobi mapping is the morphism of Hodge structures of type $(-d,-d)$ defined by the composition

$$
H^{*}(X, \mathbb{C}) \stackrel{q^{*}}{\longrightarrow} H^{*}(E, \mathbb{C}) \stackrel{p_{*}}{\longrightarrow} H^{*-2 d}(F, \mathbb{C})
$$


where $p_{*}$ the Poincaré dual of $p_{*}$ on homology when $E$ is smooth and is defined via a desingularization of $E$ when $E$ is not smooth.

With $F$ as above assume further that the generic subvariety parametrized by $F$ is smooth. The tangent space $\mathcal{T}_{F, Y}$ to $F$ at a subvariety $Y \in F$ maps naturally into $H^{0}\left(\mathcal{N}_{Y \mid X}\right)$, the tangent space to the deformation space of $Y$ in $X$. Then for generic $Y$ in $F$ there is a commutative diagram

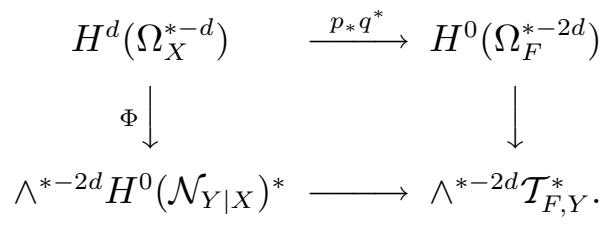

where the map in the top row is identified with the $(*-d, d)$ piece of the Abel-Jacobi mapping and $\Phi$ is the infinitesimal Abel-Jacobi mapping which is given by the contraction mapping

$$
\Phi: \wedge^{*-2 d} H^{0}\left(\mathcal{N}_{Y \mid X}\right) \otimes H^{d}\left(\Omega_{X}^{*-d}\right) \longrightarrow H^{d}\left(\Omega_{Y}^{d}\right) .
$$

When $*=2 d+1, \Phi$ also computes the differential at $Y$ of the Abel-Jacobi mapping of Griffiths from $F$ into the $(n-d) t h$ intermediate Jacobian of $X$. For details see [4].

As Abel-Jacobi mappings measure the cohomology generated by a subvariety as it deforms in a fixed variety $X$, normal functions measure the cohomology generated by a subvariety as it deforms with $X$. An infinitesimal invariant of normal functions was first introduced by Griffiths in [7] and later refined by Green in [6]. Let $S=\operatorname{Spec}\left(\mathbb{C}[s] / s^{2}\right)$. If $Y$ is an algebraic cycle of dimension $d$ on $X$ and $Y_{S}$ is an infinitesimal deformation of $Y$ in an infinitesimal deformation $X_{S}$ of $X$, then there is a natural pairing

$$
\Phi: H^{0}\left(\mathcal{N}_{Y \mid X_{S}}\right) \otimes H^{d}\left(\left.\Omega_{X_{S}}^{d+1}\right|_{X}\right) \longrightarrow H^{d}\left(\Omega_{Y}^{d}\right)
$$

given by contraction. If $\eta \in H^{0}\left(\mathcal{N}_{Y \mid X_{S}}\right)$ is the vector field determined by $Y_{S} \subseteq X_{S}$, then it is shown in Section 2 that $\Phi(\eta, \cdot)$ computes the infinitesimal invariant of the normal function associated to $Y_{S} \subseteq X_{S}$. For a related interpretation of this invariant see [15].

This pairing is similar in form to the infinitesimal Abel-Jacobi mapping and this observation is exploited to give a general technique for computing both invariants in Section 3. This construction is a generalization of a construction of Clemens in [4] and reduces the computation of $\Phi$ for certain forms on $X$ to a residue computation at a finite number of points of $Y$. In the sections that follow several applications of this technique are given. By appealing to a regularity result for space curves in [8], this technique 
is used to prove some nonvanishing results for the infinitesimal Abel-Jacobi mapping

$$
\Phi: \wedge^{n-2} H^{0}\left(\mathcal{N}_{C \mid X}\right) \otimes H^{1}\left(\Omega_{X}^{n-1}\right) \longrightarrow H^{1}\left(\Omega_{C}^{1}\right)
$$

associated to certain low degree curves $C$ on varieties $X$ of dimension $n$. In particular, it is shown that the infinitesimal Abel-Jacobi mapping is of maximal rank for low degree smooth rational curves $C$ on complete intersection threefolds $X$ with trivial canonical bundle. Combining this with the fact that the infinitesimal Abel-Jacobi mapping is trivial for such $C$ which deform generically with $X$ forces $H^{0}\left(\mathcal{N}_{C \mid X}\right)$ to vanish. This yields finiteness results for low degree rational curves on complete intersection threefolds with trivial canonical bundle. There has been a great deal of interest in this problem stemming from some computations of physicists working in string theory. The mirror symmetry principle allows them to relate the number of rational curves of a given degree on a generic quintic threefold (or more generally a Calabi-Yau threefold) to the coefficients of a certain Fourier series determined by the variation of Hodge structure of some other family of threefolds with trivial canonical bundle. It would therefore be of great interest to determine whether there was a finite number of rational curves of each degree on a generic complete intersection threefold with trivial canonical bundle. For a nice survey mirror symmetry and its implications in algebraic geometry see [13].

In the last two sections the formulas for the infinitesimal invariant of a normal function developed in Section 3 are refined in the case of subvarieties of hypersurfaces. This leads to a generalization of Voisin's formula [16] for the infinitesimal invariant of normal functions associated to algebraic one-cycles on hypersurface threefolds which are contained in a hyperplane section.

This work is an expanded version of some results in the authors dissertation completed at the University of Utah. The author would like to thank Herb Clemens for suggesting these problems and for many helpful conversations.

\section{Normal Functions.}

Let $X$ be a smooth projective variety of dimension $n$ and let $X_{S}$ be an infinitesimal deformation of $X$. Let $Y$ be an algebraic cycle on $X$ of dimension $d$. When $Y$ is smooth there is a natural pairing

$$
\Phi: H^{0}\left(\mathcal{N}_{Y \mid X_{S}}\right) \otimes H^{d}\left(\left.\Omega_{X_{S}}^{d+1}\right|_{X}\right) \longrightarrow H^{d}\left(\Omega_{Y}^{d}\right)
$$

given by contraction. When $Y$ is not smooth this pairing is still defined if we take a desingularization $f: \tilde{Y} \longrightarrow Y$ of the irreducible components of $Y$ 
and define the normal bundle to $Y$ in $X_{S}$ by $\mathcal{N}_{Y \mid X_{S}}=f^{*} \mathcal{T}_{X_{S}} / \mathcal{T}_{\tilde{Y}}$ and replace $\Omega_{Y}^{d}$ with $\Omega_{\tilde{Y}}^{d}$ in (2.1). This agrees with the usual definition of $\mathcal{N}_{Y \mid X_{S}}$ when $Y$ is smooth. Let $Y_{S} \subseteq X_{S}$ be an infinitesimal deformation of $Y$ in $X_{S}$ given by $\eta \in H^{0}\left(\mathcal{N}_{Y \mid X_{S}}\right)$. The goal of this section is to prove that:

Theorem 2.1. $\Phi(\eta, \cdot)$ computes the infinitesimal invariant of the normal function associated to $Y_{S} \subseteq X_{S}$.

The key step in this process will be the computation of $H^{d}\left(\left.\Omega_{X_{S}}^{d+1}\right|_{X}\right)$. For simplicity, assume that $S$ is one-dimensional. Then there is an exact sequence

$$
\left.0 \longrightarrow\left(\mathcal{N}_{X \mid X_{S}}\right)^{*} \longrightarrow \Omega_{X_{S}}\right|_{X} \longrightarrow \Omega_{X} \longrightarrow 0
$$

where $\left(\mathcal{N}_{X \mid X_{S}}\right)^{*}=\mathcal{I}_{X \mid X_{S}} / \mathcal{I}_{X \mid X_{S}}^{2}=\mathcal{I}_{X \mid X_{S}}=\left.\mathcal{O}_{X} \cdot d s \subseteq \Omega_{X_{S}}\right|_{X}$ and $\mathcal{I}_{X \mid X_{S}}$ is the ideal sheaf of $X$ in $X_{S}$. This induces an exact sequence

$$
\left.0 \longrightarrow \Omega_{X}^{d} \stackrel{\wedge d s}{\longrightarrow} \Omega_{X_{S}}^{d+1}\right|_{X} \longrightarrow \Omega_{X}^{d+1} \longrightarrow 0 .
$$

To obtain an explicit description of $H^{d}\left(\left.\Omega_{X_{S}}^{d+1}\right|_{X}\right)$ it will be necessary to find a nice Dolbeault type resolution of $\left.\Omega_{X_{S}}^{d+1}\right|_{X}$.

Assume that $X_{S} \longrightarrow S$ comes from a family of smooth projective varieties $\mathcal{X} \longrightarrow T$ where $T$ is a disc in $\mathbb{C}$ centered at the origin $0 \in \mathbb{C}$ and $X=X_{0}$. After shrinking $\mathrm{T}$, if necessary, there is a diffeomorphism $\Psi: X \times T \longrightarrow \mathcal{X}$. The fibers $X_{t}$ of $\mathcal{X} \longrightarrow T$ can then viewed as a family of complex structures on the $C^{\infty}$ manifold $X$. From this point of view, Kodaira in [12, Chapter 5], describes this variation of the complex structure in terms of the KodairaSpencer class of the deformation. Let $\left\{U_{k}\right\}$ be an open cover of $X$ and let $z_{k}^{1}, \ldots, z_{k}^{n}$ be local holomorphic coordinates on $U_{k}$ for $X$. Then on each $U_{k} \times T$

$$
\Psi\left(z_{k}^{1}, \ldots, z_{k}^{n}, t\right)=\left(\zeta_{k}^{1}(z, t), \ldots, \zeta_{k}^{n}(z, t), t\right)
$$

where $\zeta_{k}^{1}(z, t), \ldots, \zeta_{k}^{n}(z, t)$ are local $C^{\infty}$ coordinates on $X$ which are holomorphic with respect to the complex structure on $X_{t}$. On each $\left(U_{j} \cap U_{k}\right) \times T$, write $\zeta_{k}^{l}(z, t)=f_{j, k}^{l}\left(\zeta_{k}(z, t), t\right)$. Note that $f_{j, k}^{l}$ is holomorphic in $\zeta_{k}^{1}, \ldots, \zeta_{k}^{n}, t$ and $\zeta_{k}^{l}(z, t)$ is holomorphic in $t$ for all $\mathrm{j}, \mathrm{k}, \mathrm{l}$. Thus we can write $\zeta_{k}^{l}(z, t)$ as a power series in $t, \zeta_{k}^{l}(z, t)=z_{k}^{l}+t \alpha_{k}^{l}(z)+\cdots$. The Kodaira-Spencer class of the deformation is the image of $\partial / \partial t$ under the Kodaira-Spencer map and is represented by the $\bar{\partial}$-closed $C^{\infty}$ vector valued $(0,1)$ form $\theta$ where

$$
\left.\theta\right|_{U_{k}}=\sum \theta_{j, k}^{i} \frac{\partial}{\partial z_{k}^{i}} \otimes d \bar{z}_{k}^{j} \quad \text { and } \quad \theta_{j, k}^{i}=-\partial \alpha_{k}^{i} / \partial \bar{z}_{k}^{j} .
$$

Replacing $T$ by $S$ gives the same set up with all equations taken modulo $t^{2}$. We wish to determine the $\bar{\partial}$ operator on $X_{S}$ in terms of $z$ and $s$. First 
notice that

$$
\bar{\partial} \zeta_{k}^{l}=\bar{\partial}\left(z_{k}^{l}+s \alpha_{k}^{l}\right)=s \bar{\partial} \alpha_{k}^{l}=-s \sum \theta_{j, k}^{l} d \bar{z}_{k}^{j}=-\left.s \theta\right|_{U_{k}}\left(z_{k}^{l}\right)
$$

Thus $\left(\bar{\partial}+\left.s \theta\right|_{U_{k}}\right)\left(\zeta_{k}^{l}\right)=0$ for each $l$ and a straight forward computation shows that a $C^{\infty}$ function $f(z, s)$ on $U_{k} \times S$ is holomorphic when viewed as a function on $\Psi\left(U_{k} \times S\right)$ if and only if $\left(\bar{\partial}+\left.s \theta\right|_{U_{k}}\right)(f)=0$. Thus $\bar{\partial}+s \theta$ detects the holomorphic functions on $X_{S}$. Since $\zeta_{k}^{1}, \ldots, \zeta_{k}^{n}, s$ are local holomorphic coordinates on $U_{k} \times S \cong \Psi\left(U_{k} \times S\right),\left.\left(\left.\Omega_{X_{S}}\right|_{X}\right)\right|_{U_{k}}$ is generated by $d s$ and $d \zeta_{k}^{i}=d z_{k}^{i}+\alpha_{k}^{i} d s, i=1, \ldots, n$ over $\mathcal{O}_{U_{k}}$.

Let $\mathcal{A}^{p, q}=\mathcal{A}^{p, q}(X, \mathbb{C})$ denote the sheaf of $C^{\infty}(p, q)$-forms on X. The following lemma will enable us to effectively compute $H^{d}\left(\left.\Omega_{X_{S}}^{d+1}\right|_{X}\right)$.

Lemma 2.2. For each $p \geq 1$ there is a commutative diagram of fine resolutions

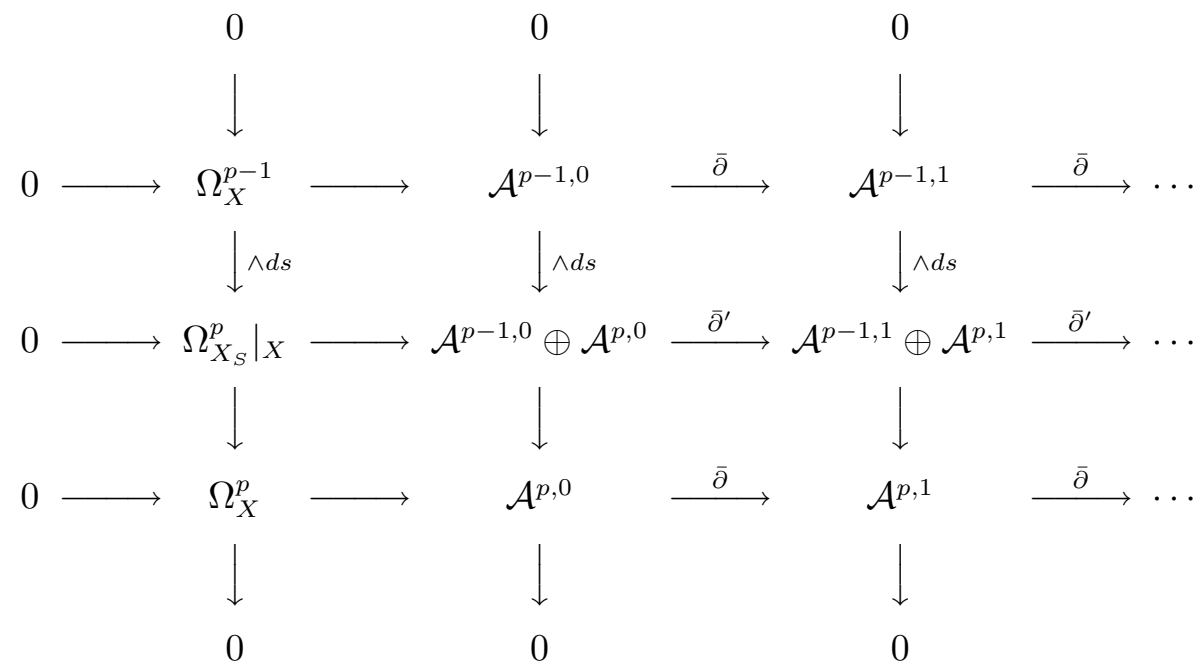

where $\bar{\partial}^{\prime}(\zeta \wedge d s+\omega)=(\bar{\partial} \zeta+\theta \cdot \omega) \wedge d s+\bar{\partial} \omega$.

Proof. The commutativity of the diagram is clear and the exactness of the top and bottom rows is Dolbeault's lemma. To complete the proof of the lemma it only remains to be shown that the middle row is exact. Using the fact that $\bar{\partial}^{2}=0$ and $\bar{\partial} \theta=0$ it is easy to check that $\bar{\partial}^{\prime} \circ \bar{\partial}^{\prime}=0$. By definition, $\bar{\partial} \alpha_{k}^{i}=-\sum \theta_{j, k}^{i} d \bar{z}_{k}^{j}$ and $\left.\theta\right|_{U_{k}}=\sum \theta_{j, k}^{i} \frac{\partial}{\partial z_{k}^{i}} \otimes d \bar{z}_{k}^{j}$ so that $\bar{\partial}^{\prime}\left(d \zeta_{k}^{i}\right)=$ $\bar{\partial}^{\prime}\left(d z_{k}^{i}+\alpha_{k}^{i} d s\right)=0$. Considering $\left.\Omega_{X_{S}}^{p}\right|_{X}$ as a subsheaf of $\mathcal{A}^{p-1,0} \wedge d s \oplus \mathcal{A}^{p, 0}$, it then follows that

$$
\left.\Omega_{X_{S}}^{p}\right|_{X} \subseteq \operatorname{ker}\left(\mathcal{A}^{p-1,0} \wedge d s \oplus \mathcal{A}^{p, 0} \stackrel{\bar{\partial}^{\prime}}{\longrightarrow} \mathcal{A}^{p-1,1} \wedge d s \oplus \mathcal{A}^{p, 1}\right) .
$$


For the opposite inclusion, let $\zeta \wedge d s+\omega \in \mathcal{A}^{p-1,0}(U) \wedge d s \oplus \mathcal{A}^{p, 0}(U)$ where $U$ is a small polydisc in $X$ and suppose $\bar{\partial}^{\prime}(\zeta \wedge d s+\omega)=0$. Then $\bar{\partial} \omega=0$ and $\bar{\partial} \zeta=-\theta \cdot \omega$ so that $\omega \in \Omega_{X}^{p}(U)$. Let $\tilde{\omega}$ be any form in $\left.\Omega_{X_{S}}^{p}\right|_{X}(U)$ which restricts to $\omega$. Then the image of $\tilde{\omega}$ in $\mathcal{A}^{p-1,0}(U) \wedge d s \oplus \mathcal{A}^{p, 0}(U)$ is $\bar{\partial}^{\prime}$-closed and of the form $\zeta^{\prime} \wedge d s+\omega$ where $\bar{\partial} \zeta^{\prime}=-\theta \cdot \omega$. It then follows that $\zeta-\zeta^{\prime} \in \Omega_{X}^{p-1}(U)$ and $\left(\zeta-\zeta^{\prime}\right) \wedge d s+\tilde{\omega}$ defines an element of $\left.\Omega_{X_{S}}^{p}\right|_{X}(U)$ whose image $\mathcal{A}^{p-1,0}(U) \wedge d s \oplus \mathcal{A}^{p, 0}(U)$ is $\zeta \wedge d s+\omega$.

Now suppose that $q \geq 1$ and $\zeta \wedge d s+\omega \in \mathcal{A}^{p-1, q}(U) \wedge d s \oplus \mathcal{A}^{p, q}(U)$ is $\bar{\partial}^{\prime}$-closed. Then $\bar{\partial} \omega=0$ and $\bar{\partial} \zeta=-\theta \cdot \omega$. By Dolbeault's lemma, there is a $\gamma \in \mathcal{A}^{p, q-1}(U)$ such that $\bar{\partial} \gamma=\omega$. Since $\bar{\partial} \theta=0, \bar{\partial} \zeta=-\theta \cdot \bar{\partial} \gamma=\bar{\partial}(\theta \cdot \gamma)$ so by Dolbeault's lemma there is a $\beta \in \mathcal{A}^{p-1, q-1}(U)$ such that $\bar{\partial} \beta=\zeta-\theta \cdot \gamma$. Putting this together one obtains

$$
\bar{\partial}^{\prime}(\beta \wedge d s+\gamma)=(\bar{\partial} \beta+\theta \cdot \gamma) \wedge d s+\bar{\partial} \gamma=\zeta \wedge d s+\omega .
$$

Thus the middle row is also exact.

Proof of Theorem 2.1. The resolutions of Lemma 2.2 compute the cohomology groups of $\Omega_{X}^{d},\left.\Omega_{X}^{d+1}\right|_{X}$ and $\Omega_{X}^{d+1}$. Furthermore, in the long exact sequence of cohomology groups for the sequence (2.2) the boundary map $H^{d}\left(\Omega_{X}^{d+1}\right) \longrightarrow H^{d+1}\left(\Omega_{X}^{d}\right)$ is given by contraction with the Kodaira-Spencer class $\theta$. Thus a cohomology class $\omega \in H^{d}\left(\Omega_{X}^{d+1}\right)$ extends to a cohomology class $\tilde{\omega} \in H^{d}\left(\left.\Omega_{X_{S}}^{d+1}\right|_{X}\right)$ if and only if $\theta \cdot \omega=0$. Lemma 2.2 also shows that in this case $\tilde{\omega}$ is represented by a form $-\zeta \wedge d s+\omega$ for some $\zeta \in A^{d, d}(X, \mathbb{C})$ with $\bar{\partial} \zeta=\theta \cdot \omega$.

Consider the exact sequence of normal bundles

$$
\left.0 \longrightarrow \mathcal{N}_{Y \mid X} \longrightarrow \mathcal{N}_{Y \mid X_{S}} \longrightarrow \mathcal{N}_{X \mid X_{S}}\right|_{Y} \longrightarrow 0 .
$$

Since $s=0$ is the defining equation for $X$ in $X_{S}$ and $s^{2}=0$, then $\left.\mathcal{N}_{X \mid X_{S}}\right|_{Y} \cong$ $\mathcal{O}_{Y} \cdot(\partial / \partial s)$. If $Y_{S}$ is an infinitesimal deformation of $Y$ in $X_{S}$ given by $\eta \in H^{0}\left(\mathcal{N}_{Y \mid X_{S}}\right)$, then the image of $\eta$ in $\left.\mathcal{N}_{X \mid X_{S}}\right|_{Y}$ corresponds to $\partial / \partial s$. In this situation, $\left.\mathcal{N}_{Y \mid X_{S}} \cong \mathcal{N}_{Y \mid X} \oplus \mathcal{N}_{X \mid X_{S}}\right|_{Y}$ since the obstruction to splitting the sequence is the image of $\partial / \partial s$ in $H^{1}\left(\mathcal{N}_{Y \mid X}\right) \cong \operatorname{Ext}^{1}\left(\mathcal{O}_{Y} \cdot(\partial / \partial s), \mathcal{N}_{Y \mid X}\right)$. Then locally $\eta=\eta^{\prime}+\partial / \partial s$ for some $\eta^{\prime} \in \mathcal{N}_{Y \mid X}$ and the pairing

$$
\Phi: H^{0}\left(\mathcal{N}_{Y \mid X_{S}}\right) \otimes H^{d}\left(\left.\Omega_{X_{S}}^{d+1}\right|_{X}\right) \longrightarrow H^{d}\left(\Omega_{Y}^{d}\right) \stackrel{\int_{Y}}{\longrightarrow} \mathbb{C}
$$

takes

$$
\eta \otimes \tilde{\omega}=\left(\eta^{\prime}+\partial / \partial s\right) \otimes(-\zeta \wedge d s+\omega) \longrightarrow-\int_{Y} \zeta+\int_{Y} \eta \cdot \omega .
$$

This is up to a sign the same as the formula given by Griffiths in [7, pp. 302-307] and completes that proof of Theorem 2.1. 


\section{Computation of the pairings.}

In this section a general method for computing both the infinitesimal AbelJacobi mapping and the infinitesimal invariant associated to a normal function will be given. As we have seen these invariants are both given by contracting normal vector fields on a subvariety $Y \subseteq X$ against forms on $X$. The computation of these invariants will be reduced to a residue computation at a finite number of points on the subvariety. This generalizes a construction of Clemens in [4] for computing the infinitesimal Abel-Jacobi mapping for curves.

Let $X$ be a smooth projective variety of dimension $n$ embedded in a smooth projective variety $W$ of dimension $m$. Let $Y$ be a smooth projective variety immersed as a subvariety of dimension $d$ in $X$ by a morphism $f: Y \longrightarrow X \subseteq W$. Let $X_{S}$ be an infinitesimal deformation of $X$ in an infinitesimal deformation $W_{S}$ of $W$. Throughout this section $S$ will denote either $\operatorname{Spec}\left(\mathbb{C}[s] / s^{2}\right)$ or $\operatorname{Spec}(\mathbb{C})$. In the later case $X_{S}$ and $W_{S}$ will be identified with $X$ and $W$ respectively. In any case, for $k \geq 2 d+1$ there is a natural pairing

$$
\Phi: \wedge^{k-2 d} H^{0}\left(\mathcal{N}_{Y \mid X_{S}}\right) \otimes H^{d}\left(\left.\Omega_{X_{S}}^{k-d}\right|_{X}\right) \longrightarrow H^{d}\left(\Omega_{Y}^{d}\right) \cong \mathbb{C}
$$

given by contraction. When $S=\operatorname{Spec}(\mathbb{C})$ this reduces to the infinitesimal Abel-Jacobi mapping for $Y$. When $S=\operatorname{Spec}\left(\mathbb{C}[s] / s^{2}\right), k=2 d+1$ and $Y$ deforms with $X$ in $X_{S}$ this is the pairing that gives the infinitesimal invariant of a normal function.

Consider the commutative diagram of exact sequences

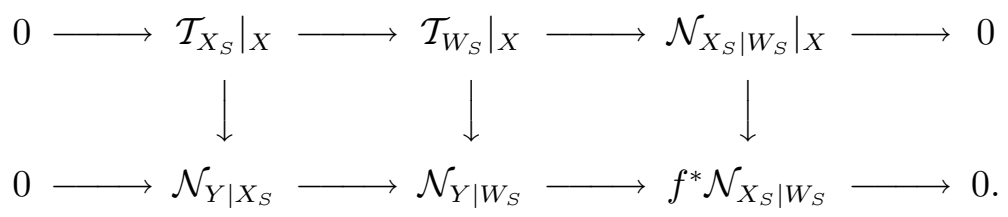

The obstruction $\mu \in \operatorname{Ext}^{1}\left(\left.\mathcal{N}_{X_{S} \mid W_{S}}\right|_{X},\left.\mathcal{T}_{X_{S}}\right|_{X}\right)$ to splitting the top row of (3.2) determines the obstruction $\sigma \in \operatorname{Ext}^{1}\left(f^{*} \mathcal{N}_{X_{S} \mid W_{S}}, \mathcal{N}_{Y \mid X_{S}}\right)$ to splitting the bottom row of (3.2) in the sense that applying the functor $\operatorname{Hom}\left(\left.\mathcal{N}_{X_{S} \mid W_{S}}\right|_{X}, \cdot\right)$ to the top row and $\operatorname{Hom}\left(f^{*} \mathcal{N}_{X_{S} \mid W_{S}}, \cdot\right)$ to the bottom row gives a commutative diagram

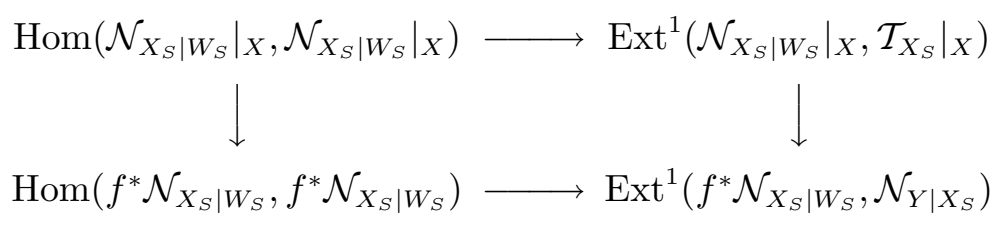


such that $\mu$ is mapped to $\sigma$.

We wish to compute $\Phi$ for forms in the image of the composition

$$
\begin{aligned}
H^{0}\left(\left.\Omega_{X_{S}}^{k} \otimes \mathcal{N}_{X_{S} \mid W_{S}}^{\otimes d}\right|_{X}\right) & \longrightarrow H^{1}\left(\left.\Omega_{X_{S}}^{k} \otimes \mathcal{T}_{X_{S}} \otimes \mathcal{N}_{X_{S} \mid W_{S}}^{\otimes(d-1)}\right|_{X}\right) \longrightarrow \cdots \\
& \longrightarrow H^{d}\left(\left.\Omega_{X_{S}}^{k} \otimes \mathcal{T}_{X_{S}}^{\otimes d}\right|_{X}\right) \longrightarrow H^{d}\left(\left.\Omega_{X_{S}}^{k-d}\right|_{X}\right)
\end{aligned}
$$

where each map

$$
H^{i}\left(\left.\Omega_{X_{S}}^{k} \otimes \mathcal{T}_{X_{S}}^{\otimes i} \otimes \mathcal{N}_{X_{S} \mid W_{S}}^{\otimes(d-i)}\right|_{X}\right) \longrightarrow H^{i+1}\left(\left.\Omega_{X_{S}}^{k} \otimes \mathcal{T}_{X_{S}}^{\otimes(i+1)} \otimes \mathcal{N}_{X_{S} \mid W_{S}}^{\otimes(d-i-1)}\right|_{X}\right)
$$

is the boundary map given by cup product with $\mu$ and the final map is given by contraction.

Similarly, there is a composition of maps

$$
\begin{aligned}
H^{0}\left(f^{*}\left(\Omega_{X_{S}}^{k} \otimes \mathcal{N}_{X_{S} \mid W_{S}}^{\otimes d}\right)\right) & \longrightarrow H^{1}\left(f^{*} \Omega_{X_{S}}^{k} \otimes \mathcal{N}_{Y \mid X_{S}} \otimes f^{*} \mathcal{N}_{X_{S} \mid W_{S}}^{\otimes d-1}\right) \longrightarrow \\
\cdots & \longrightarrow H^{d}\left(f^{*} \Omega_{X_{S}}^{k} \otimes \mathcal{N}_{Y \mid X_{S}}^{\otimes d}\right)
\end{aligned}
$$

each given by cup product with $\sigma$. The commutativity of (3.2) then gives a commutative diagram

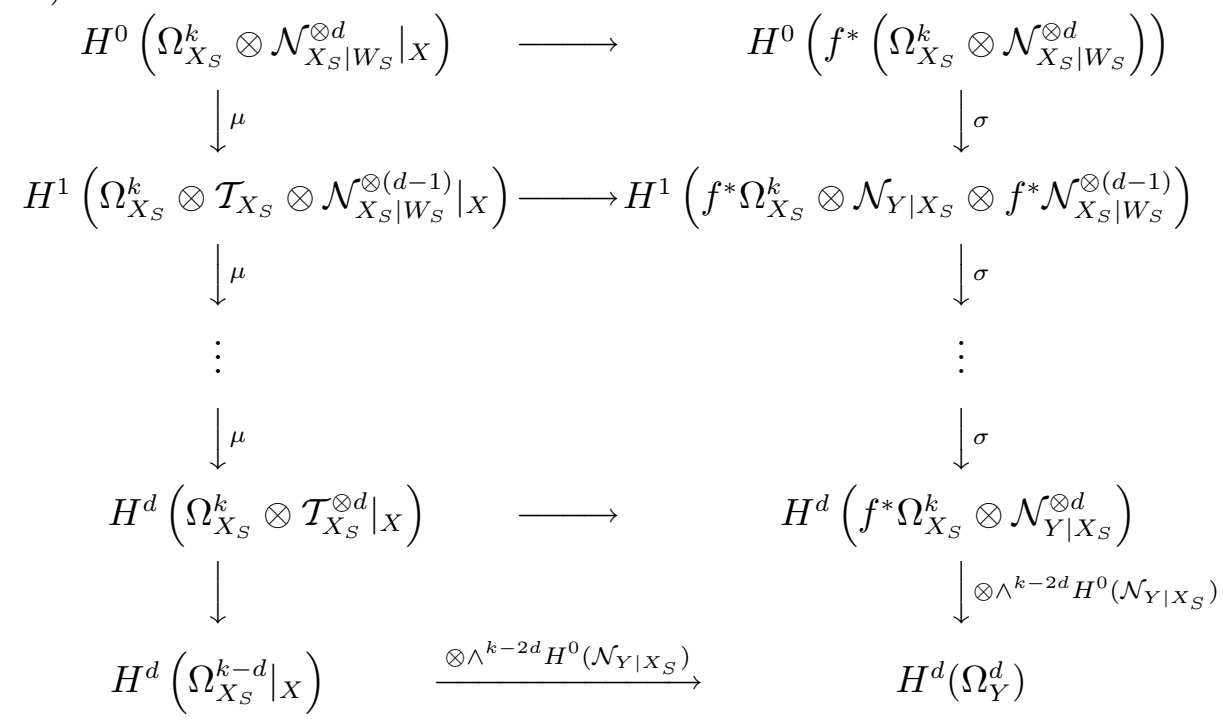

where the final horizontal and vertical maps are given by contraction. This reduces the computation of $\Phi$ for forms in the image of (3.3) to a computation involving objects defined on $Y$. In particular, this proves the following generalization of [3, Lemma 1.3].

Proposition 3.1 If $\sigma=0$ or equivalently the sequence

$$
0 \longrightarrow \mathcal{N}_{Y \mid X_{S}} \longrightarrow \mathcal{N}_{Y \mid W_{S}} \longrightarrow f^{*} \mathcal{N}_{X_{S} \mid W_{S}} \longrightarrow 0
$$


splits, then $\Phi$ vanishes on the image $H^{0}\left(\left.\Omega_{X_{S}}^{k} \otimes \mathcal{N}_{X_{S} \mid W_{S}}^{\otimes d}\right|_{X}\right)$ in $H^{d}\left(\left.\Omega_{X_{S}}^{k-d}\right|_{X}\right)$.

Let $\mathcal{L}_{1}, \ldots, \mathcal{L}_{d}$ be subline bundles of $\left.\mathcal{N}_{X_{S} \mid W_{S}}\right|_{X}$ and for each i, let $\mathcal{T}_{i} \subseteq$ $\mathcal{N}_{Y \mid W_{S}}$ denote the inverse image of $f^{*} \mathcal{L}_{i} \subseteq f^{*} \mathcal{N}_{X_{S} \mid W_{S}}$. Then there is an exact sequence

$$
0 \longrightarrow \mathcal{N}_{Y \mid X_{S}} \longrightarrow \mathcal{T}_{i} \longrightarrow f^{*} \mathcal{L}_{i} \longrightarrow 0
$$

The obstruction $\sigma$ to splitting the bottom row of (3.2) then restricts to the obstruction $\sigma_{i} \in \operatorname{Ext}^{1}\left(f^{*} \mathcal{L}_{i}, \mathcal{N}_{Y \mid X_{S}}\right)$ to splitting (3.5). This gives a commutative diagram similar to $(3.4)$ with $\mathcal{N}_{X_{S} \mid W_{S}}^{\otimes(d-i+1)}$ replaced by $\mathcal{L}_{i} \otimes \cdots \otimes \mathcal{L}_{d}$ and the vertical column on the right replaced by the composition

$$
\begin{gathered}
H^{0}\left(f^{*}\left(\Omega_{X_{S}}^{k} \otimes \mathcal{L}_{1} \otimes \cdots \otimes \mathcal{L}_{d}\right)\right) \stackrel{\sigma_{1}}{\longrightarrow} H^{1}\left(f^{*} \Omega_{X_{S}}^{k} \otimes \mathcal{N}_{Y \mid X_{S}} \otimes f^{*}\left(\mathcal{L}_{2} \otimes \cdots \otimes \mathcal{L}_{d}\right)\right) \\
\stackrel{\sigma_{2}}{\longrightarrow} \cdots \stackrel{\sigma_{d}}{\longrightarrow} H^{d}\left(f^{*} \Omega_{X_{S}}^{k} \otimes \mathcal{N}_{Y \mid X_{S}}^{\otimes d}\right) .
\end{gathered}
$$

Let $\mathcal{S}_{i}$ be a subline bundle of $\mathcal{T}_{i}$ whose image in $f^{*} \mathcal{N}_{X_{S} \mid W_{S}}$ generically generates $\mathcal{L}_{i}$ and let $D_{i}$ be the effective divisor on $Y$ given by the scheme theoretic degeneracy locus of the morphism of line bundles $\mathcal{S}_{i} \longrightarrow f^{*} \mathcal{L}_{i}$. For notational convenience, set $\mathcal{N}=\mathcal{N}_{Y \mid X_{S}}$. Let $\tilde{\mathcal{S}}_{i}=\mathcal{S}_{i}\left(D_{i}\right)$ be the sheaf of sections of $\mathcal{S}_{i}$ which are holomorphic except for poles along the components of $D_{i}$ of order not exceeding the multiplicity of the component. Let $\tilde{\mathcal{T}}_{i}$ be the subsheaf of $\mathcal{T}_{i}\left(D_{i}\right)$ generated by $\mathcal{T}_{i}$ and $\tilde{\mathcal{S}}_{i}$ and let $\tilde{\mathcal{N}}_{i}$ be the kernel of the map $\tilde{\mathcal{T}}_{i} \longrightarrow f^{*} \mathcal{L}_{i}$. Then there is a natural map

$$
f^{*} \mathcal{L}_{i} \longrightarrow \tilde{\mathcal{S}}_{i} \subseteq \tilde{\mathcal{T}}_{i}
$$

which gives the meromorphic inverse of $\mathcal{S}_{i} \longrightarrow f^{*} \mathcal{L}_{i}$ and splits the exact sequence

$$
0 \longrightarrow \tilde{\mathcal{N}}_{i} \longrightarrow \tilde{\mathcal{T}}_{i} \longrightarrow f^{*} \mathcal{L}_{i} \longrightarrow 0
$$

Let $\tau_{i} \in \operatorname{Ext}^{1}\left(\tilde{\mathcal{N}}_{i} / \mathcal{N}, \mathcal{N}\right)$ be the obstruction to splitting the exact sequence

$$
0 \longrightarrow \mathcal{N} \longrightarrow \tilde{\mathcal{N}}_{i} \longrightarrow \tilde{\mathcal{N}}_{i} / \mathcal{N} \longrightarrow 0
$$

Applying the functor $\operatorname{Hom}\left(f^{*} \mathcal{L}_{i}, \cdot\right)$ to the exact sequence (3.7) we obtain an exact sequence

$$
\begin{gathered}
\cdots \longrightarrow \operatorname{Hom}\left(f^{*} \mathcal{L}_{i}, \tilde{\mathcal{N}}_{i}\right) \longrightarrow \operatorname{Hom}\left(f^{*} \mathcal{L}_{i}, \tilde{\mathcal{N}}_{i} / \mathcal{N}\right) \stackrel{\tau_{i}}{\longrightarrow} \\
\operatorname{Ext}^{1}\left(f^{*} \mathcal{L}_{i}, \mathcal{N}\right) \longrightarrow \operatorname{Ext}^{1}\left(f^{*} \mathcal{L}_{i}, \tilde{\mathcal{N}}_{i}\right) \longrightarrow \cdots .
\end{gathered}
$$

Since the sequence $(3.6)$ splits the the obstruction $\sigma_{i} \in \operatorname{Ext}^{1}\left(f^{*} \mathcal{L}_{i}, \mathcal{N}\right)$ to splitting (3.5) goes to zero in $\operatorname{Ext}^{1}\left(f^{*} \mathcal{L}_{i}, \tilde{\mathcal{N}}_{i}\right)$. Then there is a $\sigma_{i}^{\prime} \in$ 
$\operatorname{Hom}\left(f^{*} \mathcal{L}_{i}, \tilde{\mathcal{N}}_{i} / \mathcal{N}\right)$ such that $\tau_{i} \circ \sigma_{i}^{\prime}=\sigma_{i}$. In fact, $\sigma_{i}^{\prime}$ is represented by the composition

$$
f^{*} \mathcal{L}_{i} \longrightarrow \tilde{\mathcal{T}}_{i} \longrightarrow \tilde{\mathcal{T}}_{i} / \mathcal{T}_{i} \cong \tilde{\mathcal{N}}_{i} / \mathcal{N} .
$$

Assume that the $D_{i}$ intersect properly so that $P=D_{1} \cap D_{2} \cap \cdots \cap D_{d}$ is a possibly nonreduced set of points and set

$$
\begin{aligned}
& D^{[i]}=D_{i}+\cdots+D_{d} \\
& \mathcal{L}^{[i]}=\mathcal{L}_{i} \otimes \cdots \otimes \mathcal{L}_{d} \\
& \tilde{\mathcal{N}} / \mathcal{N}^{[i]}=\tilde{\mathcal{N}}_{i} / \mathcal{N} \otimes \cdots \otimes \tilde{\mathcal{N}}_{d} / \mathcal{N}
\end{aligned}
$$

for $i=1, \ldots, d$. Then we have a commutative diagram

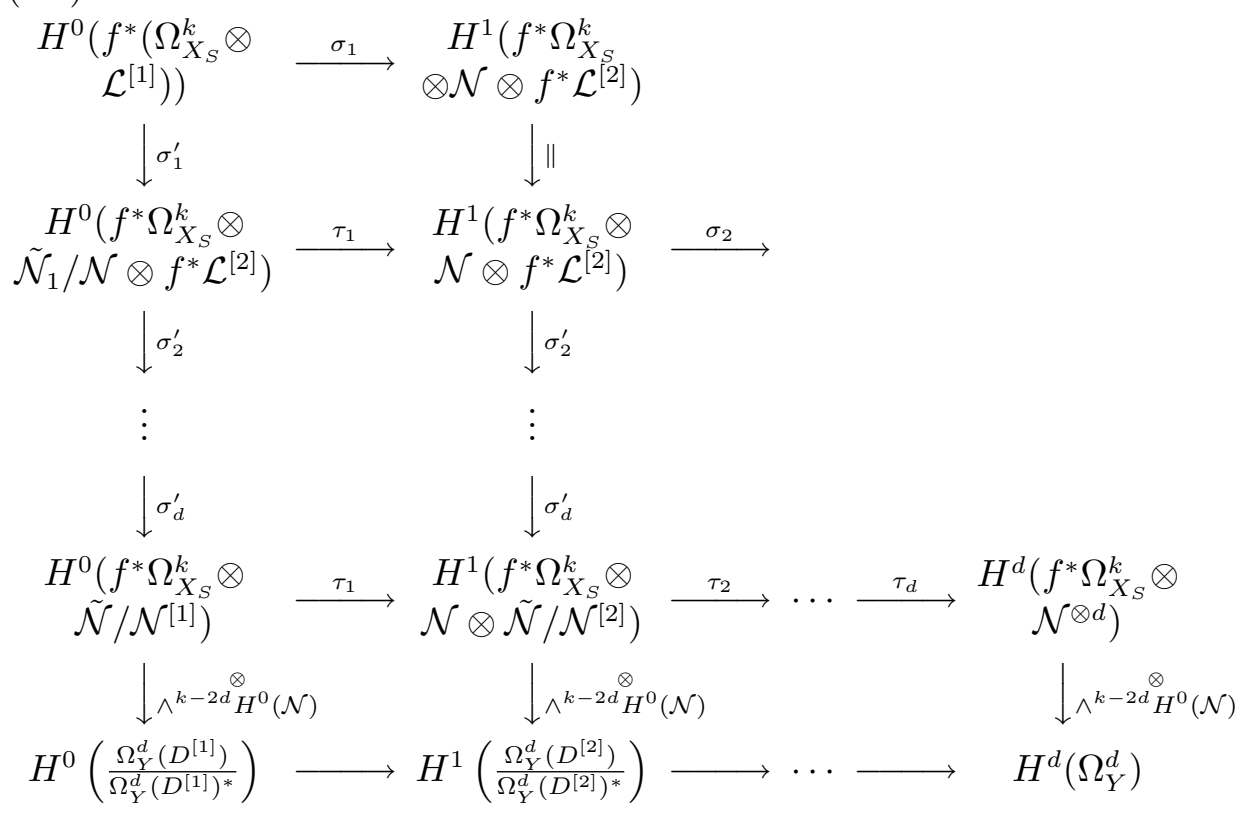

where $\Omega_{Y}^{d}\left(D^{[i]}\right)$ is the sheaf of meromorphic $d$-forms on $Y$ which are allowed to have poles along the divisors $D_{i}, \ldots, D_{d}$ but are otherwise holomorphic and $\Omega_{Y}^{d}\left(D^{[i]}\right)^{*}$ is the subsheaf of $\Omega_{Y}^{d}\left(D^{[i]}\right)$ consisting of those forms which are holomorphic along at least one of the divisors $D_{i}, \ldots, D_{d}$. The bottom row of (3.8) can then be identified with the usual residue mapping for meromorphic $d$-forms on $Y$ at the points of $P$

$$
H^{0}\left(\frac{\Omega_{Y}^{d}\left(\sum_{i=1}^{d} D_{i}\right)}{\sum_{j=1}^{d} \Omega_{Y}^{d}\left(\sum_{i \neq j} D_{i}\right)}\right) \stackrel{\operatorname{Res}_{P}}{\longrightarrow} \mathbb{C} .
$$


For $i=1, \ldots, d$, let $s_{i}$ be a nontrivial meromorphic section of $\mathcal{S}_{i}$ and let $\bar{s}_{i}$ be the image of $s_{i}$ in $f^{*} \mathcal{L}_{i}$. Then it follows that $\sigma_{i}^{\prime} \in \operatorname{Hom}\left(f^{*} \mathcal{L}_{i}, \tilde{\mathcal{N}}_{i} / \mathcal{N}\right)$ is represented by

$$
s_{i} / \bar{s}_{i} \in \operatorname{Hom}\left(f^{*} \mathcal{L}_{i}, \tilde{\mathcal{S}}_{i}\right) \subseteq \operatorname{Hom}\left(f^{*} \mathcal{L}_{i}, \tilde{\mathcal{T}}_{i}\right)
$$

If $\omega \in H^{d}\left(\left.\Omega_{X_{S}}^{k-d}\right|_{X}\right)$ is the image of $\tilde{\omega} \in H^{0}\left(\left.\Omega_{X_{S}}^{k}\right|_{X} \otimes \mathcal{L}_{1} \otimes \cdots \otimes \mathcal{L}_{d}\right)$ under the map given by (3.3) and $\eta \in \wedge^{k-2 d} H^{0}\left(\mathcal{N}_{Y \mid X_{S}}\right)$ then combining the above results we find that

$$
\Phi(\eta, \omega)=\sum \operatorname{Res}_{p}\left(\frac{\eta \wedge s_{d} \wedge \cdots \wedge s_{1}}{\bar{s}_{1} \cdots \bar{s}_{d}} \rightarrow f^{*} \tilde{\omega}\right)
$$

where the sum is taken over all points $p$ in the support of $P=D_{1} \cap D_{2} \cap$ $\cdots \cap D_{d}$.

A geometric method of obtaining such a setup in the case when $X_{S}$ has codimension one in $W_{S}$ is to take $d$ subvarieties $Z_{i} \subseteq W_{S}$ each containing $Y$ in its smooth locus such that $\operatorname{dim} Z_{i}=\operatorname{dim} Y+1$ and the intersections $Z_{i} \cdot X_{S}=Y+Y_{i}$ have the property that $Y_{i}$ intersects $Y$ properly along a divisor $D_{i}$ whose support is contained in the smooth locus of $Y$. If $\mathcal{N}_{Y \mid Z_{i}}$ is invertible for each $\mathrm{i}$, then in the above construction we can take $\mathcal{L}_{i}=$ $\left.\mathcal{N}_{X_{S} \mid W_{S}}\right|_{X}, \mathcal{T}_{i}=\mathcal{N}_{Y \mid W_{S}}$ and $\mathcal{S}_{i}=\mathcal{N}_{Y \mid Z_{i}}$ for each i. If $\mathcal{N}_{Y \mid Z_{i}}$ is not invertible then $\mathcal{S}_{i}$ can be taken to be any invertible subsheaf of $\mathcal{N}_{Y \mid Z_{i}}$. This construction also works if the $Z_{i}$ are only defined in a first order neighborhood of $Y$ in $W_{S}$ which amounts to choosing $d$ global sections $s_{i}$ of $\mathcal{N}_{Y \mid W_{S}}$ and taking $\mathcal{S}_{i}$ to be the subline bundle of $\mathcal{N}_{Y \mid W_{S}}$ generated by $s_{i}$. In fact, it was these examples that initially motivated the above construction.

In the case when $S=\operatorname{Spec}(\mathbb{C}), k=n$ and the pairing $\Phi$ given by (3.1) is the usual infinitesimal Abel-Jacobi mapping we can produce sections of $\Omega_{X}^{n}$ via the isomorphism

$$
\Omega_{W}^{m} \otimes \wedge^{m-n} \mathcal{N}_{X \mid W} \longrightarrow \Omega_{X}^{n}
$$

This isomorphism is given locally as follows. Let $\rho$ be a meromorphic section of $\wedge^{m-n} \mathcal{T}_{W}$ whose image $\bar{\rho}$ in $\wedge^{m-n} \mathcal{N}_{X \mid W}$ is not zero. Then the isomorphism given by (3.10) can be viewed as contraction with $\rho / \bar{\rho}$. If we choose a different $\rho$, then the results of the two contraction will agree where they are both defined. This gives a convenient way to produce local representatives of forms in $H^{0}\left(\Omega_{X}^{n}\right)$. Similarly, local representatives of forms in $H^{0}\left(f^{*}\left(\Omega_{X}^{n}\right)\right)$ can be obtained by contracting sections of $H^{0}\left(f^{*}\left(\Omega_{W}^{m} \otimes \wedge^{m-n} \mathcal{N}_{X \mid W}\right)\right)$ against $\rho / \bar{\rho}$ where $\rho$ is now any meromorphic section of $\wedge^{m-n} f^{*} \mathcal{T}_{W}$ whose image $\bar{\rho}$ 
in $\wedge^{m-n} f^{*} \mathcal{N}_{X \mid W}$ is not zero. Consider the commutative diagram

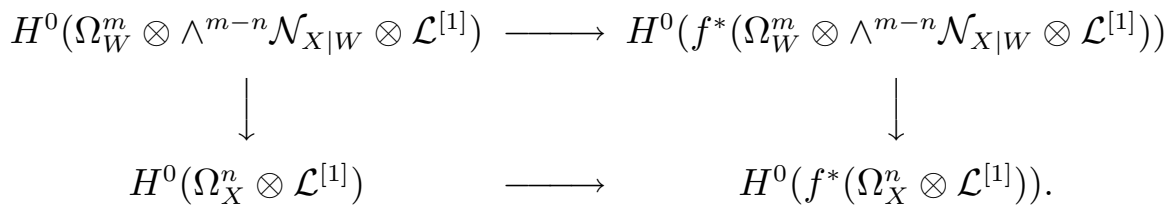

If $\omega \in H^{d}\left(\Omega_{X}^{n-d}\right)$ is the image of $\tilde{\omega} \in H^{0}\left(\Omega_{W}^{m} \otimes \wedge^{m-n} \mathcal{N}_{X \mid W} \otimes \mathcal{L}^{[1]}\right)$ under the composition

$(3.11) H^{0}\left(\Omega_{W}^{m} \otimes \wedge^{m-n} \mathcal{N}_{X \mid W} \otimes \mathcal{L}^{[1]}\right) \longrightarrow H^{0}\left(\Omega_{X}^{n} \otimes \mathcal{L}^{[1]}\right) \longrightarrow H^{d}\left(\Omega_{X}^{n-d}\right)$

and $\eta \in \wedge^{n-2 d} H^{0}\left(\mathcal{N}_{Y \mid X}\right)$ then (3.9) can be rewritten

$$
\Phi(\eta, \omega)=\sum \operatorname{Res}_{p}\left(\frac{\eta \wedge s_{d} \wedge \cdots \wedge s_{1} \wedge \rho}{\bar{s}_{1} \cdots \bar{s}_{d} \bar{\rho}} \rightarrow f^{*} \tilde{\omega}\right)
$$

where the $s_{i}$ and $\bar{s}_{i}$ are defined as before and $\rho$ is any meromorphic section of $\wedge^{m-n} \mathcal{N}_{Y \mid W}=\wedge^{m-n}\left(f^{*} \mathcal{T}_{W} / \mathcal{T}_{Y}\right)$ whose image $\bar{\rho}$ in $\wedge^{m-n} f^{*} \mathcal{N}_{X \mid W}$ does not vanish at any of the points $p \in P$.

\section{Nonvanishing results for $K_{X}$ nef.}

In this section we will use the results of Section 3 to prove some nonvanishing results for the infinitesimal Abel-Jacobi mapping for low degree curves on projective varieties $X$ with nef canonical bundle $K_{X}$. These results depend on the regularity theorem for space curves of Gruson, Lazarsfeld and Peskine in [8]. Let $\omega$ denote the natural map $\wedge^{n-2} H^{0}\left(\mathcal{N}_{C \mid X}\right) \longrightarrow H^{0}\left(\wedge^{n-2} \mathcal{N}_{C \mid X}\right)$.

Theorem 4.1. $\quad$ Let $X \subseteq \mathbb{P}^{m}$ be a smooth projective variety of dimension $n$ with $K_{X}$ nef and let $f: C \longrightarrow X \subseteq \mathbb{P}^{m}$ be a smooth curve of genus $g$ and degree $d$ on $X$ with $\operatorname{deg}\left(\mathcal{N}_{C \mid X}\right)<0$. Assume that $X$ is a divisor on a smooth variety $Z \subseteq \mathbb{P}^{m}$ such that $\mathcal{N}_{C \mid Z}$ is generated by global sections and $\Omega_{X}^{n} \otimes \mathcal{N}_{X \mid Z} \cong \mathcal{O}_{X}(k)$ for some positive integer $k$ with $d \leq k+2$. Then the infinitesimal Abel-Jacobi mapping $\Phi: \wedge^{n-2} H^{0}\left(\mathcal{N}_{C \mid X}\right) / \operatorname{ker} \omega \longrightarrow H^{1}\left(\Omega_{X}^{n-1}\right)^{*}$ is injective.

Proof. In the construction of Section 3 take $\mathcal{L}=\mathcal{N}_{X \mid Z} \subseteq \mathcal{N}_{X \mid \mathbb{P} m}$. Then (3.5) is replaced by the exact sequence

$$
0 \longrightarrow \mathcal{N}_{C \mid X} \longrightarrow \mathcal{N}_{C \mid Z} \longrightarrow f^{*} \mathcal{N}_{X \mid Z} \longrightarrow 0
$$

By our hypothesis, $H^{0}\left(\Omega_{\mathbb{P} m}^{m} \otimes \wedge^{m-n} \mathcal{N}_{X \mid \mathbb{P}^{m}} \otimes \mathcal{N}_{X \mid Z}\right) \cong H^{0}\left(\Omega_{X}^{n} \otimes \mathcal{N}_{X \mid Z}\right) \cong$ $H^{0}\left(\mathcal{O}_{X}(k)\right)$ and thus can be identified with a quotient of

$$
\left\{G \Omega: G \in S^{k}\right\}
$$


where $S^{k}$ is the set of homogeneous polynomials of degree $k$ on $\mathbb{P}^{m}$ and

$$
\Omega=\sum(-1)^{j} x_{j} d x_{0} \wedge \cdots \wedge \widehat{d x_{j}} \wedge \cdots \wedge d x_{m}
$$

is a nonzero global section of $\Omega_{\mathbb{P} m}^{m}(m+1) \cong \mathcal{O}_{\mathbb{P}^{m}}$. Denote by $\omega_{G}$ the image of $G \Omega$ in $H^{1}\left(\Omega_{X}^{n-1}\right)$ under the mapping defined by (3.11) for each $G \in S^{k}$.

Fix an $\eta \in \wedge^{n-2} H^{0}\left(\mathcal{N}_{C \mid X}\right)$ whose image in $H^{0}\left(\wedge^{n-2} \mathcal{N}_{C \mid X}\right)$ is nonzero. By our hypothesis, $\operatorname{deg}\left(\mathcal{N}_{C \mid X}\right)<0$ so that $\mathcal{N}_{C \mid X}$ cannot be semipositive. If we denote by $\mathcal{N}_{C \mid X}^{+}$the subsheaf of $\mathcal{N}_{C \mid X}$ generated by its global sections, then $\mathcal{N}_{C \mid Z}$ is semipositive implies that the exact sequence

$$
0 \longrightarrow \mathcal{N}_{C \mid X} / \mathcal{N}_{C \mid X}^{+} \longrightarrow \mathcal{N}_{C \mid Z} / \mathcal{N}_{C \mid X}^{+} \longrightarrow f^{*} \mathcal{N}_{X \mid Z} \longrightarrow 0
$$

cannot split. In this situation, there is a global section $s \in H^{0}\left(\mathcal{N}_{C \mid Z}\right)$ whose image $\bar{s} \in H^{0}\left(f^{*} \mathcal{N}_{X \mid Z}\right)$ is not zero and a simple point $p_{1}$ in the zero locus of $\bar{s}$ such that $\eta \wedge s$ does not vanish at $p_{1}$. To see this pick $s$ so that $\bar{s}$ has distinct zeros and does not vanish at any of the zeros of $\eta$. Since (4.1) does not split there is a point $p_{1}$ in the zero locus of $\bar{s}$ such that $s$ is not zero in the geometric fiber of $\left(\mathcal{N}_{C \mid Z} / \mathcal{N}_{C \mid X}^{+}\right)$at $p_{1}$. Since $\eta \in \wedge^{n-2} H^{0}\left(\mathcal{N}_{C \mid X}^{+}\right)$does not vanish at $p_{1}$, then $\eta \wedge s$ will not vanish at $p_{1}$. Let $l=\operatorname{deg}\left(f^{*} \mathcal{N}_{X \mid Z}\right)$ and let $p_{2}, \ldots, p_{l}$ be the other zeros of $\bar{s}$. Let $\rho$ be any global section of $\wedge^{m-n} \mathcal{N}_{C \mid \mathbb{P}^{m}}$ whose image $\bar{\rho}$ in $\wedge^{m-n} f^{*} \mathcal{N}_{X \mid \mathbb{P}^{m}}$ does not vanish at any of the $p_{j}$. Then (3.12) can be rewritten

$$
\Phi\left(\eta, \omega_{G}\right)=\sum \operatorname{Res}_{p_{j}}\left(\frac{\eta \wedge s \wedge \rho}{\bar{s} \bar{\rho}} \rightarrow f^{*}(G \Omega)\right)
$$

for any $G \in S^{k}$. By construction $\eta \wedge s \wedge \rho$ defines a global section of $\wedge^{m-1} \mathcal{N}_{C \mid \mathbb{P}^{m}}$ which does not vanish at $p_{1}$.

Thus to complete the proof of the theorem we need to show that it is possible to pick $G \in S^{k}$ such that $G$ vanishes at every $p_{j}$, except $p_{1}$ and does not vanish at $p_{1}$. Since $C$ is smooth of degree $d \leq k+2$, then by the main result of [8] the natural map

$$
H^{0}\left(\mathcal{O}_{\mathbb{P}}(k)\right) \longrightarrow H^{0}\left(\mathcal{O}_{C}(k)\right)
$$

is surjective. Since $\operatorname{deg}\left(\mathcal{N}_{C \mid X}\right)=-K_{X} \cdot C+2 g-2<0$, then $K_{X} \cdot C \geq$ $2 g-1$. Also note that by the definitions of $k$ and $l, K_{X} \cdot C=d k-l$. Thus $d k-(l-1) \geq 2 g$. Since $S^{k}$ cuts out a complete linear system on $C$ of degree $d k$ and $d k-(l-1) \geq 2 g$, then it follows from the Riemann-Roch theorem that we can choose $G$ to vanish at any $l-1$ points of $C$ and not vanish at $p_{1}$.

Let $X \subseteq \mathbb{P}^{m}$ be a complete intersection of $m-n$ hypersurfaces $Y_{1}, \ldots$, $Y_{m-n}$ in $\mathbb{P}^{m}$. For each $j$, let $k_{j}$ denote the degree of $Y_{j}$ and let $Z_{j}=\cap_{i \neq j} Y_{i}$. 
Then $X$ has dimension $n$ and when $X$ is smooth the canonical bundle of $X$ is isomorphic to $\mathcal{O}\left(\sum k_{j}-m-1\right)$. We call such an $X$ a complete intersection of type $\left(k_{1}, \ldots, k_{m-n}\right)$ in $\mathbb{P}^{m}$. For complete intersections, Theorem 4.1 has the following form:

Corollary 4.2. $\quad$ Let $X$ be a generic complete intersection of type $\left(k_{1}, \ldots\right.$, $\left.k_{m-n}\right)$ in $\mathbb{P}^{m}$ such that $K_{X}$ is nef or equivalently $\sum k_{j} \geq m+1$. Let $f: C \longrightarrow$ $X \subseteq \mathbb{P}^{m}$ be a smooth curve of genus $g$ and degree $d$ on $X$ and assume there is an $i$ such that $d \leq \sum k_{j}-m+1+k_{i}, d\left(m+1-\sum k_{j}\right)+2 g-2<0$ and $\mathcal{N}_{C \mid Z_{i}}$ is generated by global sections. Then $\Phi: \wedge^{n-2} H^{0}\left(\mathcal{N}_{C \mid X}\right) / \operatorname{ker} \omega \longrightarrow H^{1}\left(\Omega_{X}^{n-1}\right)^{*}$ is injective.

Proof. Since $X$ is a generic complete intersection we may assume that $Z_{i}$ is smooth. Then the $\operatorname{deg}\left(\mathcal{N}_{X \mid Z_{i}}\right)=k_{i}$ and $\Omega_{X}^{n} \otimes \mathcal{N}_{X \mid Z_{i}} \cong \mathcal{O}_{X}\left(\sum k_{j}-m-1+k_{i}\right)$. Also notice that $\operatorname{deg}\left(\mathcal{N}_{C \mid X}\right)=-K_{X} \cdot C+2 g-2=d\left(m+1-\sum k_{j}\right)+2 g-2<0$ so that all the hypotheses of Theorem 4.1 are satisfied.

Remark. When the canonical bundle of $X$ is not necessarily nef the basic argument of Theorem 4.1 will still go through with some modification. For example, it can be shown that if $X$ is a smooth hypersurface of degree $m$ in $\mathbb{P}^{m}, m \geq 4$ and $f: C \longrightarrow X \subseteq \mathbb{P}^{m}$ is a smooth rational curve of degree $d \leq m+1$ such that the global sections of $\mathcal{N}_{C \mid X}$ generate a subsheaf of rank $\geq m-3$ then the infinitesimal Abel-Jacobi mapping is nonzero. For this and related results see [17].

\section{Rational curves on $K$-trivial complete intersection threefolds.}

Let $C$ be a smooth curve on a smooth threefold $X$. Then there is an exact sequence

$$
H^{0}\left(\mathcal{T}_{X}\right) \longrightarrow H^{0}\left(\mathcal{N}_{C \mid X}\right) \longrightarrow \mathcal{H}^{1} \longrightarrow H^{1}\left(\mathcal{T}_{X}\right) \stackrel{\phi}{\longrightarrow} H^{1}\left(\mathcal{N}_{C \mid X}\right)
$$

where $\mathcal{H}^{1}$ is the first hypercohomology group of the map $\mathcal{T}_{X} \longrightarrow \mathcal{N}_{C \mid X}$ and classifies the first order infinitesimal deformations of the pair $(C, X)$. When $C$ is rational and $X$ has trivial canonical bundle, the map $\phi$ can be identified with the infinitesimal Abel-Jacobi mapping via the commutative diagram

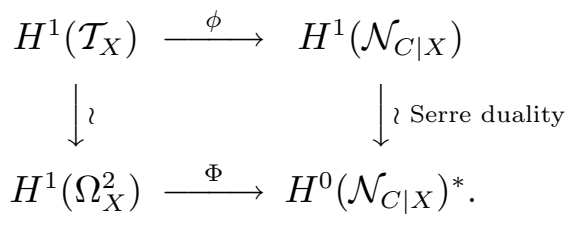


When $C$ deforms generically with $X$ to first order the mapping $\mathcal{H}^{1} \longrightarrow$ $H^{1}\left(\mathcal{T}_{X}\right)$ of $(5.1)$ is surjective and the infinitesimal Abel-Jacobi mapping must vanish. This gives the following vanishing result.

Lemma 5.1. Let $X$ be a smooth threefold with trivial canonical bundle. If $C$ is a smooth rational curve on $X$, then $C$ deforms generically with $X$ to first order if and only if the infinitesimal Abel-Jacobi mapping is zero.

If $X$ is a smooth complete intersection threefold with trivial canonical bundle, then it is easy to check that $X$ is a complete intersection of type (5), $(2,4),(3,3),(3,2,2)$, or $(2,2,2,2)$. For the generic $(5)$ and $(2,4)$ complete intersection, the $Z_{i}$ in Corollary 4.2 can be taken to be $\mathbb{P}^{4}$ and a smooth quadric fourfold respectively. Then $\mathcal{N}_{C \mid Z}$ is then generated by global sections since $Z_{i}$ is a homogeneous space. For the remaining cases the arguments of [5, Lecture 21] show that when $C$ deforms generically with $X$ there is an $i$ such that $\mathcal{N}_{C \mid Z_{i}}$ is generated by global sections. Combining this with Lemma 5.1 and Corollary 4.2 gives the following finiteness result for smooth rational curves on such $X$.

Theorem 5.2. $\quad$ Let $X$ be a generic complete intersection threefold with trivial canonical bundle. Then $X$ has only a finite number of smooth rational curves of degree $d$ if

(i) $d \leq 7$ and $X$ is of type (5)

(ii) $d \leq 6$ and $X$ is of type $(2,4)$

(iii) $d \leq 5$ and $X$ is of type $(3,3)$

(iv) $d \leq 4$ and $X$ is of type $(2,2,3)$ or $(2,2,2,2)$.

This result in the case of quintic threefolds was originally proven by Katz in [11] and recently strengthened by Johnsen and Kleiman in [10], and Nijsse in [14] to $d \leq 9$. A similar result for complete intersection threefolds was also obtained independently by Huybrechts in [9]. Huybrechts method only yields the result for $d \leq 4$ in the $(2,4)$ complete intersection case.

Let $S$ be the moduli space of all smooth complete intersection threefolds of one of the types $(5),(2,4),(3,3),(2,2,3)$, or $(2,2,2,2)$ and let $S_{d, a}$ denote the set of smooth threefolds $X$ in $S$ which admit a smooth rational curve of degree $d$ with normal bundle $\mathcal{N}_{C \mid X} \cong \mathcal{O}(a) \oplus \mathcal{O}(-a-2)$. Then $S_{d, a}$ is a locally closed, possibly empty, subvariety of $S$ for each $d$ and $a \geq-1$.

Theorem 5.3. If $d$ is as in the statement of Theorem 5.2, then the codimension of $S_{d, a}$ in $S$ is $\geq a+1$. 
Proof. Let $X$ be a smooth threefold in $S_{d, a}$ and let $C$ be a smooth rational curve on $X$ with $\mathcal{N}_{C \mid X} \cong \mathcal{O}(a) \oplus \mathcal{O}(-a-2)$. By Theorem 4.1, for $d$ in the above range the infinitesimal Abel-Jacobi mapping $\Phi: H^{0}\left(\mathcal{N}_{C \mid X}\right) \longrightarrow$ $H^{1}\left(\Omega_{X}^{2}\right)^{*}$ is injective. Then its dual $\phi$ is surjective and since $H^{0}\left(\mathcal{T}_{X}\right)=0$ the sequence

$$
0 \longrightarrow H^{0}\left(\mathcal{N}_{C \mid X}\right) \longrightarrow \mathcal{H}^{1} \longrightarrow H^{1}\left(\mathcal{T}_{X}\right) \stackrel{\phi}{\longrightarrow} H^{1}\left(\mathcal{N}_{C \mid X}\right) \longrightarrow 0
$$

is exact. Since $H^{1}\left(\mathcal{N}_{C \mid X}\right) \cong H^{0}\left(\mathcal{N}_{C \mid X}\right)$ and so has dimension $a+1$ the image of $\mathcal{H}^{1}$ in $H^{1}\left(\mathcal{T}_{X}\right)$ has codimension $a+1$. Thus $C$ deforms to first order over a codimension $a+1$ subset of the tangent space to $X$ in $S$. Since the rank of $H^{0}\left(\mathcal{N}_{C \mid X}\right)$ can only drop as $C$ moves generically with $X$, this shows that $S_{d, a}$ has codimension $\geq a+1$ in $S$.

These results will also be valid for low degree rational curves on any smooth threefold $X$ with trivial canonical bundle if one can show that $X$ is a divisor in some smooth variety $Z$ satisfying the hypothesis of Theorem 4.1.

\section{Some computations for hypersurfaces}

In this section we will apply the results of Section 3 to obtain some formulas for the infinitesimal invariant of normal functions associated to subvarieties of hypersurfaces. There is an added difficulty in applying the results of Section 3 in the case when $S=\operatorname{Spec}\left(\mathbb{C}[s] / s^{2}\right)$ in that the cohomology groups $H^{i}\left(\left.\Omega_{X_{S}}^{n-i}\right|_{X}\right)$ are more difficult to compute. Let $X$ be a smooth hypersurface of degree $m$ in $\mathbb{P}^{n+1}, n \geq 3$ defined by a homogeneous polynomial $F$. Then $F+s G$ defines an infinitesimal deformation $X_{G} \subseteq \mathbb{P}_{S}^{n+1}$ of $X$ for any homogeneous polynomial $G$ of degree $m$. Let $x_{0}, \ldots, x_{n+1}$ be homogeneous coordinates for $\mathbb{P}^{n+1}$ and let $F_{i}$ denote the partial derivative $\partial F / \partial x_{i}$ for $i=0, \ldots, n+1$. Set:

$S=$ the graded ring $\mathbb{C}\left[x_{0}, \ldots, x_{n+1}\right] /(F)$

$J=$ the homogeneous ideal generated by $F_{0}, \ldots, F_{n+1}$

$R=S / J$, the Jacobian ring of $F$

$S^{a}, J^{a}, R^{a}=$ the ath graded piece of $S, J, R$ respectively. Let $\Omega=\Omega_{\mathbb{P}^{n+1}}=\sum(-1)^{i} x_{i} d x_{0} \wedge \cdots \wedge \overline{d x_{i}} \wedge \cdots \wedge d x_{n+1}$ and for each $J=$ $\left(i_{0}, \ldots, i_{a}\right), i_{j} \in\{0, \ldots, n+1\}$, let $\Omega_{J}=\partial / \partial x_{i_{a}} \rightarrow \cdots \rightarrow \partial / \partial x_{i_{0}} \rightarrow \Omega$ and $F_{J}=F_{i_{0}} \cdots F_{i_{a}}$. Then $\Omega$ is a nonzero global section of $\Omega_{\mathbb{P}^{n+1}}^{n+1}(n+2) \cong \mathcal{O}_{\mathbb{P}^{n+1}}$ and since $X$ is smooth $\mathcal{U}=\left\{U_{i}\right\}$ where $U_{i}=\left\{F_{i} \neq 0\right\}$ is an open cover $X$. In [1], it is shown that $R^{(a+1) m-n-2}$ can be identified with the primitive Cech cohomology group $H^{a}\left(\mathcal{U}, \Omega_{X}^{n-a}\right)^{\circ}=\check{H}^{a}\left(\Omega_{X}^{n-a}\right)^{\circ}$ via the map

$$
H \in R^{(a+1) m-n-2} \longrightarrow \omega_{H}=\left\{H \Omega_{J} / F_{J}\right\}_{|J|=a+1} .
$$


Lemma 6.1. The sections of $\check{H}^{0}\left(\Omega_{X_{G}}^{n} \otimes \mathcal{O}_{X}(d m)\right) / \check{H}^{0}\left(\Omega_{X}^{n-1} \otimes \mathcal{O}_{X}(d m)\right)$ are in one to one correspondence with

$$
\left\{H \in R^{(d+1) m-n-2}: G H=0 \text { in } R^{(d+2) m-n-2}\right\} .
$$

Furthermore, if $G H=\sum K_{j} F_{j}$ for some $K_{j} \in S^{(d+1) m-n-1}$, then

$$
\left\{\frac{H \Omega_{i}-\sum K_{j} \Omega_{j, i} \wedge d s}{F_{i}}\right\}
$$

defines a Čech 0-cycle in $\check{H}^{0}\left(\Omega_{X_{G}}^{n} \otimes \mathcal{O}_{X}(d m)\right)$ corresponding to $H$.

Proof. In the long exact sequence in cohomology for the exact sequence

$$
\left.0 \longrightarrow \Omega_{X}^{n-1} \otimes \mathcal{O}(d m) \stackrel{\wedge d s}{\longrightarrow} \Omega_{X_{G}}^{n}\right|_{X} \otimes \mathcal{O}(d m) \longrightarrow \Omega_{X}^{n} \otimes \mathcal{O}(d m) \longrightarrow 0,
$$

the boundary map $\check{H}^{0}\left(\Omega_{X}^{n} \otimes \mathcal{O}(d m)\right) \longrightarrow \check{H}^{1}\left(\Omega_{X}^{n-1} \otimes \mathcal{O}(d m)\right)$ takes $\left\{H \Omega_{i} / F_{i}\right\}$ to the 1-cocycle $\left\{G H \Omega_{i, j} / F_{i} F_{j}\right\}$. As in [1], $\left\{G H \Omega_{i, j} / F_{i} F_{j}\right\}=0$ in $\check{H}^{1}\left(\Omega_{X}^{n-1} \otimes\right.$ $\mathcal{O}(d m))$ if and only if $G H \in J^{(d+2) m-n-2}$. Now suppose $G H=\sum K_{j} F_{j} \in$ $J^{(d+2) m-n-2}$. It follows from the identity

$$
0=d F \wedge\left(\Omega_{l, i, j} \wedge d s\right)=F_{j} \Omega_{l, i} \wedge d s-F_{i} \Omega_{l, j} \wedge d s+F_{l} \Omega_{i, j} \wedge d s
$$

that

$$
\begin{aligned}
\delta\left\{\frac{\sum K_{l} \Omega_{l, i} \wedge d s}{F_{i}}\right\} & =\left\{\frac{K_{l}\left(F_{i} \Omega_{l, j}-F_{j} \Omega_{l, i}\right) \wedge d s}{F_{i} F_{j}}\right\}=\left\{\frac{\sum K_{l} F_{l} \Omega_{i, j} \wedge d s}{F_{i} F_{j}}\right\} \\
& =\left\{\frac{G H \Omega_{i, j} \wedge d s}{F_{i} F_{j}}\right\}=\delta\left\{\frac{H \Omega_{i}}{F_{i}}\right\} .
\end{aligned}
$$

Thus $\left\{\left(H \Omega_{i}-\sum K_{j} \Omega_{j, i} \wedge d s\right) / F_{i}\right\}$ defines a Čech cocycle in $\check{H}^{0}\left(\Omega_{X_{G}}^{n} \otimes\right.$ $\left.\mathcal{O}_{X}(d m)\right)$.

Let $Y$ be a smooth variety of dimension $d$ immersed as a subvariety of $X$ by a morphism $f: Y \longrightarrow X$. If $H \in S^{(d+1) m-n-2}$ and $G H=\sum K_{j} F_{j} \in$ $J^{(d+2) m-n-2}$, then by Lemma 6.1, $\left\{\left(H \Omega_{i}-\sum K_{j} \Omega_{j, i} \wedge d s\right) / F_{i}\right\}$ defines a Čech 0-cycle in $\check{H}^{0}\left(\Omega_{X_{G}}^{n} \otimes \mathcal{O}_{X}(d m)\right) \cong H^{0}\left(\left.\Omega_{X_{G}}^{n} \otimes \mathcal{N}_{X_{G} \mid \mathbb{P}_{S}^{n+1}}^{\otimes d}\right|_{X}\right)$. Denote by $\omega_{H, K}$. the image of this Cech 0 -cycle in $H^{d}\left(\left.\Omega_{X_{G}}^{n-d}\right|_{X}\right)$. We wish to compute

$$
\Phi: \wedge^{n-2 d} H^{0}\left(\mathcal{N}_{Y \mid X_{G}}\right) \otimes H^{d}\left(\left.\Omega_{X_{G}}^{n-d}\right|_{X}\right) \longrightarrow H^{d}\left(\Omega_{Y}^{d}\right)
$$

for such forms $\omega_{H, K}$. Let $s_{1}, \ldots, s_{d}$ be global sections of $\mathcal{N}_{Y \mid \mathbb{P}_{S}^{n+1}}$ whose images $\bar{s}_{1}, \ldots, \bar{s}_{d} \in H^{0}\left(f^{*} \mathcal{N}_{X_{G} \mid \mathbb{P}_{S}^{n+1}}\right)$ have the property that the zero loci $D_{i}$ 
of $\bar{s}_{i}$ intersect transversly at a reduced set of points $P$. Then by (3.9), if $\eta \in \wedge^{n-2 d} H^{0}\left(\mathcal{N}_{Y \mid X_{G}}\right)$

$$
\Phi\left(\eta, \omega_{H, K .}\right)=\sum \operatorname{Res}_{p}\left(\frac{\eta \wedge s_{d} \wedge \cdots \wedge s_{1}}{\bar{s}_{1} \cdots \bar{s}_{d}} \rightarrow f^{*}\left(\frac{H \Omega_{i}-\sum K_{j} \Omega_{j, i} \wedge d s}{F_{i}}\right)\right)
$$

where the sum is taken over all points $p \in P$.

Consider the exact sequences

$$
\begin{gathered}
0 \longrightarrow \mathcal{T}_{Y} \longrightarrow f^{*} \mathcal{T}_{\mathbb{P}_{S}^{n+1}} \longrightarrow \mathcal{N}_{Y \mid \mathbb{P}_{S}^{n+1}} \longrightarrow 0 \\
0 \longrightarrow \mathcal{O}_{Y} \longrightarrow f^{*} \mathcal{O}_{\mathbb{P}^{n+1}}(1)^{n+2} \oplus \mathcal{O}_{Y} \longrightarrow f^{*} \mathcal{T}_{\mathbb{P}_{S}^{n+1}} \longrightarrow 0
\end{gathered}
$$

If $\beta_{0}, \ldots, \beta_{n+1} \in H^{0}\left(f^{*} \mathcal{O}_{\mathbb{P}^{n+1}}(1)\right)$ and $c \in H^{0}\left(\mathcal{O}_{Y}\right)=\mathbb{C}$, then the above exact sequences show that $s=\sum \beta_{j} \partial / \partial x_{j}+c \partial / \partial s$ defines a section of $H^{0}\left(\mathcal{N}_{Y \mid \mathbb{P}_{S}^{n+1}}\right)$. Let $V$ denote the subspace of $H^{0}\left(\mathcal{N}_{Y \mid \mathbb{P}_{S}^{n+1}}\right)$ given by all such $s$. It follows that $s=\sum \beta_{j} \partial / \partial x_{j}+c \partial / \partial s \in H^{0}\left(\mathcal{N}_{Y \mid X_{G}}\right)$ if and only if the image of $s$ in $H^{0}\left(f^{*} \mathcal{N}_{X_{G} \mid \mathbb{P}_{S}^{n+1}}\right)$ is zero or equivalently $\sum \beta_{j} f^{*} F_{j}+c f^{*} G=0$ when considered as an element of the graded ring $A=\oplus_{k} H^{0}\left(f^{*} \mathcal{O}_{\mathbb{P}^{n+1}}(k)\right)$. For notational convenience we will set $g=f^{*} G, f_{i}=f^{*} F_{i}$, etc.

Notice that if we take $\rho=\partial / \partial x_{i}$, then

$$
\left(\frac{\rho}{\bar{\rho}}\right) \rightarrow f^{*}\left(H \Omega-\sum K_{j} \Omega_{j} \wedge d s\right)=f^{*}\left(\frac{H \Omega_{i}-\sum K_{j} \Omega_{j, i} \wedge d s}{F_{i}}\right) .
$$

If $\rho=\sum \beta_{j} \partial / \partial x_{j}+c \partial / \partial s \in V \cap H^{0}\left(\mathcal{N}_{Y \mid X_{G}}\right)$ and $g \neq 0$, then it can be verified that $\rho \rightarrow f^{*}\left(H \Omega-\sum K_{j} \Omega_{j} \wedge d s\right)=0$. As in Section 3 it then follows that if $\rho \in V \cap H^{0}\left(\mathcal{N}_{Y \mid \mathbb{P}_{S}^{n+1}}\right)$ and the image $\bar{\rho}$ of $\rho$ in $H^{0}\left(f^{*} \mathcal{N}_{X_{G} \mid \mathbb{P}_{S}^{n+1}}\right)$ is nonzero, then $\rho / \bar{\rho} \rightarrow f^{*}\left(H \Omega-\sum K_{j} \Omega_{j} \wedge d s\right)$ is a local representative of

$$
\left\{\frac{H \Omega_{i}-\sum K_{j} \Omega_{j, i} \wedge d s}{F_{i}}\right\} \in \check{H}^{0}\left(\Omega_{X_{G}}^{n} \otimes \mathcal{O}_{X}(d m)\right) .
$$

Thus we may take $\rho=\partial / \partial s$ and rewrite (6.1) as

$$
\Phi\left(\eta, \omega_{H, K .}\right)=\sum \operatorname{Res}_{p}\left(\frac{\left(\eta \wedge s_{d} \wedge \cdots \wedge s_{1} \wedge \sum k_{j} \partial / \partial x_{j}\right) \rightarrow f^{*} \Omega}{g \bar{s}_{1} \cdots \bar{s}_{d}}\right)
$$

whenever $\bar{\rho}=g$ does not vanish at any of the points $p \in P$.

The formula (6.2) has a rather nice interpretation when $Y=C$ is a rational curve on a hypersurface threefold $X$. In this case $C$ is defined by a morphism

$$
f([a, b])=\left[\alpha_{0}(a, b), \ldots, \alpha_{4}(a, b)\right]
$$


where each $\alpha_{i}$ is a homogeneous polynomial of degree $d$ in $a, b$, the homogeneous coordinates for $\mathbb{P}^{1}$. A variant of an argument in [2] for the reduced case shows that in this case $H^{0}\left(\mathcal{N}_{C \mid \mathbb{P}_{S}^{4}}\right)$ can be identified with

$$
\frac{\left\{\sum \beta_{i} \partial / \partial x_{i}+c \partial / \partial s: \beta_{i} \in H^{0}\left(\mathcal{O}_{\mathbb{P}^{1}}(d)\right), c \in \mathbb{C}\right\}}{\left\{\sum\left(l\left(\alpha_{i}\right)_{a}+l^{\prime}\left(\alpha_{i}\right)_{b}\right) \partial / \partial x_{i}: l, l^{\prime} \in H^{0}\left(\mathcal{O}_{\mathbb{P}^{1}}(1)\right)\right\}}
$$

where $\left(\alpha_{i}\right)_{a}$ and $\left(\alpha_{i}\right)_{b}$ are the partial derivatives of $\alpha_{i}$ with respect to $a$ and $b$ respectively. If $\eta \in H^{0}\left(\mathcal{N}_{C \mid X_{G}}\right)$, then $\eta=\sum \gamma_{i} \partial / \partial x_{i}+\partial / \partial s$ for some $\gamma_{0}, \ldots, \gamma_{4} \in H^{0}\left(\mathcal{O}_{\mathbb{P}^{1}}(d)\right)$ satisfying $\sum \gamma_{i} f_{i}=-g$. If $s=\sum \beta_{i} \partial / \partial x_{i}$, then

$$
\begin{aligned}
& \left(\eta \wedge \sum \beta_{j} \partial / \partial x_{j} \wedge \sum k_{l} \partial / \partial x_{l}\right) \rightarrow f^{*} \Omega \\
& =\sum \gamma_{i} \beta_{j} k_{l} \partial / \partial x_{i} \rightarrow \partial / \partial x_{j} \rightarrow \partial / \partial x_{l} \rightarrow f^{*} \Omega \\
& =\sum_{i, j, l} \sum_{m<n} \operatorname{sign}(i j l m n) \gamma_{i} \beta_{j} k_{l}\left(\alpha_{m} d \alpha_{n}-\alpha_{n} d \alpha_{m}\right) \\
& =(1 / d) \sum_{i, j, l} \sum_{m<n} \operatorname{sign}(i j l m n) \gamma_{i} \beta_{j} k_{l}\left(\left(\alpha_{m}\right)_{b}\left(\alpha_{n}\right)_{a}-\left(\alpha_{n}\right)_{b}\left(\alpha_{m}\right)_{a}\right)(b d a-a d b) \\
& =(1 / d) \operatorname{det}(M)(b d a-a d b)
\end{aligned}
$$

where $M$ is the $5 \times 5$ matrix

$$
M=\left(\begin{array}{ccc}
\gamma_{0} & \cdots & \gamma_{4} \\
\beta_{0} & \cdots & \beta_{4} \\
k_{0} & \cdots & k_{4} \\
\left(\alpha_{0}\right)_{b} & \cdots & \left(\alpha_{4}\right)_{b} \\
\left(\alpha_{0}\right)_{a} & \cdots & \left(\alpha_{4}\right)_{a}
\end{array}\right) .
$$

Then (6.2) can be rewritten as

$$
\Phi\left(\eta, \omega_{H, K .}\right)=(1 / d) \sum \operatorname{Res}_{p}\left(\frac{\operatorname{det}(M)(b d a-a d b)}{g\left(\sum \beta_{j} f_{j}+c g\right)}\right)
$$

where the $p$ are the distinct zeros of $g$. Similar formulas can be derived for the infinitesimal Abel-Jacobi mapping for rational curves on hypersurfaces.

\section{Some reduction results after Voisin}

Let $X$ be a smooth hypersurface of degree $m$ in $\mathbb{P}^{n+1}$ defined by a homogeneous polynomial $F$ and for each homogeneous polynomial $G$ of degree $m$ let $X_{G} \subseteq \mathbb{P}_{S}^{n+1}$ be the infinitesimal deformation of $X$ defined by $F+s G$. Let $Y$ be a smooth variety of dimension $d$ immersed as a subvariety of $X$ by a morphism $f: Y \longrightarrow X$. Assume further that the image of $Y$ is contained 
in a smooth hyperplane section $Z=X \cap \mathbb{P}^{n}$ of $X$. By a change of coordinates for $\mathbb{P}^{n+1}$, we may assume that $\mathbb{P}^{n}=\left\{x_{n+1}=0\right\}$. Denote by $F^{\prime}, G^{\prime}$ the restrictions of $F, G$ to $\mathbb{P}^{n}$. If $Y$ deforms to first order with $X$ in $X_{G}$ and remains in a hyperplane section, then after a change of coordinates for $\mathbb{P}_{S}^{n+1}$ we may assume that the deformation of $Y$ lies in $Z_{G^{\prime}} \subseteq \mathbb{P}_{S}^{n}$, where $Z_{G^{\prime}}$ is defined by $F^{\prime}+s G^{\prime}$.

In [16], Voisin shows that when $X$ is a threefold and $Y$ is an algebraic one-cycle which deforms with $X$ in a fixed hyperplane, the infinitesimal invariant of the resulting normal function can be related to the infinitesimal Abel-Jacobi mapping for $Y \subseteq Z=X \cap \mathbb{P}^{3}$. The following theorem is a generalization of this result for arbitrary $n$ and $d$ with $n \geq 2 d+1$.

Theorem 7.1. With the above assumptions and notation, let $\eta \in$ $\wedge^{n-2 d-1} H^{0}\left(\mathcal{N}_{Y \mid Z}\right)$ and assume $\eta^{\prime} \in H^{0}\left(\mathcal{N}_{Y \mid Z_{G^{\prime}}}\right)$ represents the deformation of $Y$ in $Z_{G^{\prime}}$. If $H$ is a homogeneous polynomial of degree $m(d+1)-n-2$ and $G H=\sum K_{j} F_{j}$ for some homogeneous polynomials $K_{j}$, then

$$
\Phi_{X}\left(\eta \wedge \eta^{\prime}, \omega_{H, K .}\right)=(-1)^{n+d+1} \Phi_{Z}\left(\eta, \omega_{K_{n+1}^{\prime}}\right)
$$

where $K_{n+1}^{\prime}$ is the restriction of $K_{n+1}$ to $\mathbb{P}^{n}$ and $\omega_{H, K} \in H^{d}\left(\left.\Omega_{X_{G}}^{n-d}\right|_{X}\right)$ and $\omega_{K_{n+1}^{\prime}} \in H^{d}\left(\Omega_{Z}^{n-d-1}\right)$ are defined as in Section 6.

Proof. By (6.2)

$$
\begin{aligned}
& \Phi_{X}\left(\eta \wedge \eta^{\prime}, \omega_{H, K .}\right) \\
& =\sum \operatorname{Res}_{p \in P}\left(\frac{\left(\eta \wedge \eta^{\prime} \wedge s_{d} \wedge \cdots \wedge s_{1} \wedge \sum k_{j} \partial / \partial x_{j}\right) \rightarrow f^{*} \Omega_{\mathbb{P}^{n+1}}}{g \bar{s}_{1} \cdots \bar{s}_{d}}\right)
\end{aligned}
$$

where $g=f^{*} G$ and $s_{1}, \ldots, s_{d}$ are any global sections of $\mathcal{N}_{Y \mid \mathbb{P}_{S}^{n+1}}$ whose images $\bar{s}_{1}, \ldots, \bar{s}_{d} \in H^{0}\left(f^{*} \mathcal{N}_{X_{G} \mid \mathbb{P}_{S}^{n+1}}\right)$ have the property that the zero loci of the $\bar{s}_{i}$ intersect transversly at a reduced set of points $P$. If we choose $s_{1}, \ldots, s_{d} \in H^{0}\left(\mathcal{N}_{Y \mid \mathbb{P}_{S}^{n}}\right)$, then $\eta, \eta^{\prime}, s_{1}, \ldots, s_{d}$ are all sections of $\mathcal{N}_{Y \mid \mathbb{P}_{S}^{n}}$ and it follows that

$$
\begin{aligned}
& \left(\eta \wedge \eta^{\prime} \wedge s_{d} \wedge \cdots \wedge s_{1} \wedge \sum k_{j} \partial / \partial x_{j}\right) \rightarrow f^{*} \Omega_{\mathbb{P}^{n+1}} \\
& =\left(\eta \wedge \eta^{\prime} \wedge s_{d} \wedge \cdots \wedge s_{1}\right) \rightarrow f^{*}\left(K_{n+1} \partial / \partial x_{n+1} \rightarrow \Omega_{\mathbb{P} n+1}\right) .
\end{aligned}
$$


Consider the commutative diagram

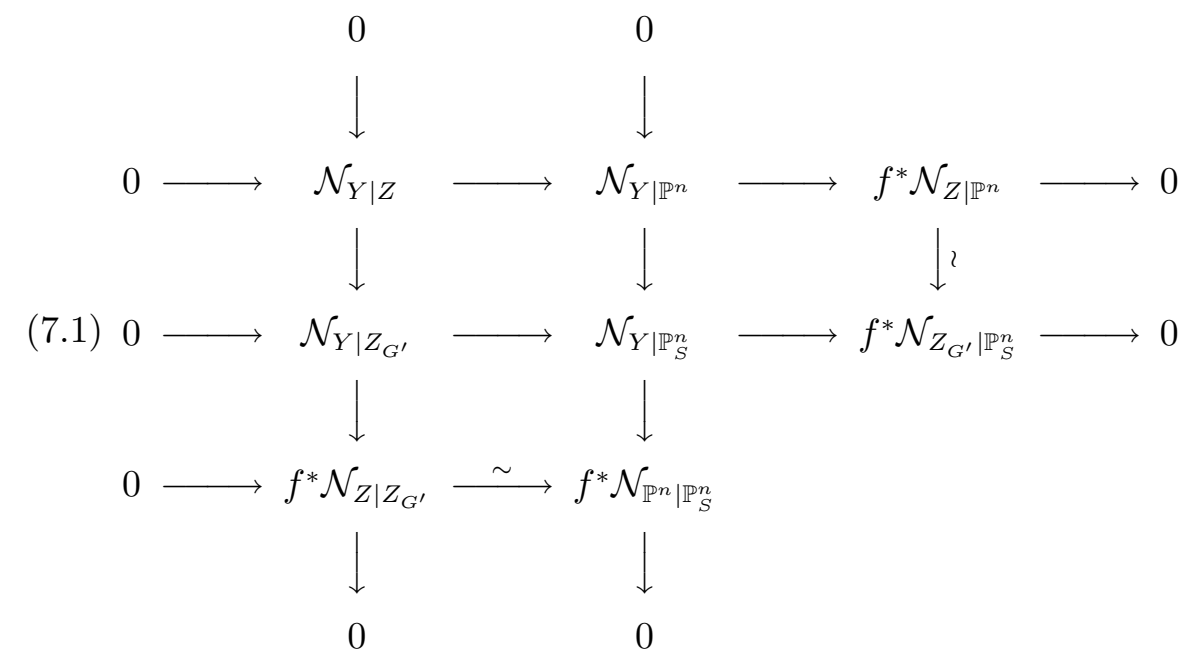

Since $Y$ deforms with $X$ in $X_{G}$, then as in the proof of Theorem 2.1 the two vertical exact sequences in $(7.1)$ split. Referring to $(7.1), \eta^{\prime} \in H^{0}\left(\mathcal{N}_{Y \mid Z_{G^{\prime}}}\right)$ has image $\partial / \partial s \in H^{0}\left(f^{*} \mathcal{N}_{\mathbb{P}^{n} \mid \mathbb{P}_{S}^{n}}\right)$. Since the image of $\partial / \partial s$ in $H^{0}\left(f^{*} \mathcal{N}_{Z_{G^{\prime}} \mid \mathbb{P}_{S}^{n}}\right)$ is $g=f^{*} G$ and the image of $\eta^{\prime}$ in $H^{0}\left(f^{*} \mathcal{N}_{Z_{G^{\prime}} \mid \mathbb{P}_{S}^{n}}\right)$ is zero, then it follows that the image of $\eta^{\prime}$ under the composition

$$
H^{0}\left(\mathcal{N}_{Y \mid Z_{G^{\prime}}}\right) \longrightarrow H^{0}\left(\mathcal{N}_{Y \mid \mathbb{P}_{S}^{n}}\right) \longrightarrow H^{0}\left(\mathcal{N}_{Y \mid \mathbb{P}^{n}}\right) \longrightarrow H^{0}\left(f^{*} \mathcal{N}_{Z \mid \mathbb{P}^{n}}\right)
$$

is $-g$. If we denote by $\rho$ the image of $\eta^{\prime}$ in $H^{0}\left(\mathcal{N}_{Y \mid \mathbb{P}^{n}}\right)$, then the image $\bar{\rho}$ of $\rho$ in $H^{0}\left(f^{*} \mathcal{N}_{Z \mid \mathbb{P}^{n}}\right)$ will be $-g$. Also note that $\partial / \partial x_{n+1} \rightarrow \Omega_{\mathbb{P}^{n+1}}=(-1)^{n} \Omega_{\mathbb{P}^{n}}$. Thus

$$
\begin{aligned}
& \Phi_{X}\left(\eta \wedge \eta^{\prime}, \omega_{H, K .}\right) \\
& =\sum \operatorname{Res}_{p \in P}\left(\frac{\left(\eta \wedge \eta^{\prime} \wedge s_{d} \wedge \cdots \wedge s_{1} \wedge \sum k_{j} \partial / \partial x_{j}\right) \rightarrow f^{*} \Omega_{\mathbb{P}^{n+1}}}{g \bar{s}_{1} \cdots \bar{s}_{d}}\right) \\
& =\sum \operatorname{Res}_{p \in P}\left(\frac{\left(\eta \wedge \eta^{\prime} \wedge s_{d} \wedge \cdots \wedge s_{1}\right) \rightarrow f^{*}\left(K_{n+1} \partial / \partial x_{n+1} \rightarrow \Omega_{\mathbb{P}^{n+1}}\right)}{g \bar{s}_{1} \cdots \bar{s}_{d}}\right) \\
& =(-1)^{n+d+1} \sum \operatorname{Res}_{p \in P}\left(\frac{\left(\eta \wedge s_{d} \wedge \cdots \wedge s_{1} \wedge \rho\right) \rightarrow f^{*}\left(K_{n+1} \Omega_{\mathbb{P}^{n}}\right)}{\bar{s}_{1} \cdots \bar{s}_{d} \bar{\rho}}\right) \\
& =(-1)^{n+d+1} \Phi_{Z}\left(\eta, \omega_{K_{n+1}^{\prime}}\right)
\end{aligned}
$$

by (3.12).

When $n=2 d+1$ the infinitesimal Abel-Jacobi mapping for $f: Y \longrightarrow Z$ is given by pullback

$$
\Phi_{Z}: H^{d}\left(\Omega_{Z}^{d}\right) \stackrel{f^{*}}{\longrightarrow} H^{d}\left(\Omega_{Y}^{d}\right) \stackrel{\int_{Y}}{\longrightarrow} \mathbb{C} .
$$


In this case Theorem 7.1 can be rewritten as

Corollary 7.2 If $n=2 d+1$ then with the notation of Theorem 7.1,

$$
\Phi_{X}\left(\eta^{\prime}, \omega_{H, K .}\right)=(-1)^{d} \int_{Y} f^{*} \omega_{K_{n+1}^{\prime}} .
$$

There are similar results for the infinitesimal Abel-Jacobi mapping associated to subvarieties of hypersurfaces which are contained in a hyperplane section. With the notation introduced earlier, assume that $Y$ is contained in a smooth hyperplane section $Z=X \cap \mathbb{P}^{n}$ where $\mathbb{P}^{n}=\left\{x_{n+1}=0\right\}$. Assume that $Y$ deforms to first order with $Z$ in $X$ or equivalently there is an $\eta^{\prime} \in H^{0}\left(\mathcal{N}_{Y \mid X}\right)$ whose image in $H^{0}\left(f^{*} \mathcal{N}_{Z \mid X}\right)$ is $f^{*} L$ for some $L \in H^{0}\left(\mathcal{N}_{Z \mid X}\right)=$ $H^{0}\left(\mathcal{O}_{Z}(1)\right)$.

Theorem 7.3 With the above assumptions and notation, let $\eta \in$ $\wedge^{n-2 d-1} H^{0}\left(\mathcal{N}_{Y \mid Z}\right)$ and let $\eta^{\prime} \in H^{0}\left(\mathcal{N}_{Y \mid X}\right)$ be as above. If $H$ is a homogeneous polynomial of degree $m(d+1)-n-2$, then

$$
\Phi_{X}\left(\eta \wedge \eta^{\prime}, \omega_{H}\right)=(-1)^{n+d+1} \Phi_{Z}\left(\eta, \omega_{L H^{\prime}}\right)
$$

where $H^{\prime}$ is the restriction of $H$ to $\mathbb{P}^{n}$ and $\omega_{H} \in H^{d}\left(\Omega_{X}^{n-d}\right)$ and $\omega_{L H^{\prime}} \in$ $H^{d}\left(\Omega_{Z}^{n-d-1}\right)$ are defined as in Section 6.

Proof. By (3.12)

$$
\Phi_{X}\left(\eta \wedge \eta^{\prime}, \omega_{H}\right)=\sum \operatorname{Res}_{p \in P}\left(\frac{\left(\eta \wedge \eta^{\prime} \wedge s_{d} \wedge \cdots \wedge s_{1} \wedge \rho\right) \rightarrow f^{*}\left(H \Omega_{\mathbb{P}^{n+1}}\right)}{\bar{s}_{1} \cdots \bar{s}_{d} \bar{\rho}}\right)
$$

where $s_{1}, \ldots, s_{d}, \rho$ are any global sections of $\mathcal{N}_{Y \mid \mathbb{P}^{n+1}}$ whose images $\bar{s}_{1}, \ldots$, $\bar{s}_{d}, \bar{\rho} \in H^{0}\left(f^{*} \mathcal{N}_{X \mid \mathbb{P}^{n+1}}\right)$ have the property that the zero loci of the $\bar{s}_{i}$ intersect transversly at a reduced set of points $P$ and $\bar{\rho}$ does not vanish at any $p \in P$. As in the proof of Theorem 7.1 we may choose $s_{1}, \ldots, s_{d}, \bar{\rho} \in H^{0}\left(\mathcal{N}_{Y \mid \mathbb{P}^{n}}\right)$, so that $\eta, s_{1}, \ldots, s_{d}, \rho$ are all sections of $\mathcal{N}_{Y \mid \mathbb{P}^{n}}$. Then by our assumption on $\eta^{\prime}$ and the fact that $\partial / \partial x_{n+1} \rightarrow \Omega_{\mathbb{P}^{n+1}}=(-1)^{n} \Omega_{\mathbb{P}^{n}}$

$$
\begin{aligned}
& \left(\eta \wedge \eta^{\prime} \wedge s_{d} \wedge \cdots \wedge s_{1} \wedge \rho\right) \rightarrow f^{*}\left(H \Omega_{\mathbb{P}^{n+1}}\right) \\
& =(-1)^{d+1}\left(\eta \wedge s_{d} \wedge \cdots \wedge s_{1} \wedge \rho\right) \rightarrow f^{*}\left(L H \partial / \partial x_{n+1} \rightarrow \Omega_{\mathbb{P}^{n+1}}\right) \\
& =(-1)^{n+d+1}\left(\eta \wedge s_{d} \wedge \cdots \wedge s_{1} \wedge \rho\right) \rightarrow f^{*}\left(L H \Omega_{\mathbb{P}^{n}}\right) .
\end{aligned}
$$

Thus

$$
\begin{aligned}
& \Phi_{X}\left(\eta \wedge \eta^{\prime}, \omega_{H}\right) \\
& (-1)^{n+d+1} \sum \operatorname{Res}_{p \in P}\left(\frac{\left(\eta \wedge s_{d} \wedge \cdots \wedge s_{1} \wedge \rho\right) \rightarrow f^{*}\left(L H \Omega_{\mathbb{P}^{n}}\right)}{\bar{s}_{1} \cdots \bar{s}_{d} \bar{\rho}}\right) \\
& =(-1)^{n+d+1} \Phi_{Z}\left(\eta, \omega_{L H^{\prime}}\right)
\end{aligned}
$$


by (3.12).

\section{References}

[1] J. Carlson and P. Griffiths, Infinitesimal variations of Hodge structure and the global Torelli problem, Journees de geometrie algebrique d'Angers, (1980), 51-76.

[2] H. Clemens, Homological equivalence modulo algebraic equivalence, is not finitely generated, Publ. Math. I.H.E.S., 58 (1983), 231-250.

[3] The local geometry of the Abel-Jacobi mapping, Proc. of Sym. in Pure Math., 46 (1987), 223-233.

[4] , The infinitesimal Abel-Jacobi mapping and the $\mathcal{O}(2)+\mathcal{O}(-4)$ curve, Duke Math. J., 59(1) (1989), 233-240.

[5] H. Clemens, J. Kollár and S. Mori, Higher dimensional complex geometry, Asterisque, 166 (1988).

[6] M. Green, Griffiths' infinitesimal invariant and the Abel-Jacobi map, J. Differential Geometry, 29 (1989), 545-555.

[7] P. Griffiths, Infinitesimal variations of Hodge structure (III): Determinantal varieties and the infinitesimal invariant of normal functions, Comp. Math., 50 (1983), 267-324.

[8] L. Gruson, R. Lazarsfeld and C. Peskine, On a theorem of Castelnuovo and the equations defining space curves, Inv. Math., 72 (1983), 491-506.

[9] D. Huybrechts, The tangent bundle of a Calabi-Yau manifold - Deformations and restriction to rational curves, Commun. Math. Phys., 171 (1995), 139-158.

[10] T. Johnsen and S. Kleiman, Rational curves of degree at most 9 on a general quintic threefold, preprint, 1995.

[11] S. Katz, On the finiteness of rational curves on quintic threefolds, Comp. Math., 60 (1986), 151-162.

[12] K. Kodaira, Complex Manifolds and Deformation of Complex Structures, Vol. 283 in 'Grundlehren der mathematischen Wissenschaften', Springer-Verlag, New York, 1986.

[13] D.R. Morrison, Mirror symmetry and rational curves on quintic threefolds: A guide for mathematicians, J. of AMS, 6 (1993), 223-247.

[14] P. Nijsse, Clemens' conjecture for octic and nonic curves, Preprint, U. Leiden, 1993.

[15] C. Voisin, Une remarque sur l'invariant infinitésimal des fonctions normales, C.R. Acad. Sci. Paris, 307 (1988), 157-160.

[16] Une approche infinitésimale du théorème de H. Clemens sur les cycles d'une quintique générale de $\mathbb{P}^{4}$, J. Alg. Geom., 1 (1992), 157-174.

[17] R. Westhoff, Curves and normal functions on higher dimensional complex varieties, Ph.D. Thesis, U. Utah, 1993.

Received August 6, 1996.

Bemidji State University

BEMIDJI, MN 56601

E-mail address: westhoff@vax1.bemidji.msus.edu 